ESAIM: COCV 20 (2014) 460-487

DOI: $10.1051 / \mathrm{cocv} / 2013071$
ESAIM: Control, Optimisation and Calculus of Variations

www.esaim-cocv.org

\title{
REGULARITY RESULTS FOR AN OPTIMAL DESIGN PROBLEM WITH A VOLUME CONSTRAINT
}

\author{
Menita Carozza ${ }^{1}$, Irene Fonseca ${ }^{2}$ and Antonia Passarelli di Napoli ${ }^{3}$
}

\begin{abstract}
Regularity results for minimal configurations of variational problems involving both bulk and surface energies and subject to a volume constraint are established. The bulk energies are convex functions with $p$-power growth, but are otherwise not subjected to any further structure conditions. For a minimal configuration $(u, E)$, Hölder continuity of the function $u$ is proved as well as partial regularity of the boundary of the minimal set $E$. Moreover, full regularity of the boundary of the minimal set is obtained under suitable closeness assumptions on the eigenvalues of the bulk energies.
\end{abstract}

Mathematics Subject Classification. 49N15, 49N60, 49N99.

Received May 29, 2013.

Published online March 7, 2014.

\section{INTRODUCTION AND STATEMENTS}

In this paper we study minimal energy configurations of a mixture of two materials in a bounded, connected open set $\Omega \subset \mathbb{R}^{n}$, when the perimeter of the interface between the materials is penalized. Precisely, the energy is given by

$$
\mathcal{I}(u, E):=\int_{\Omega}\left(F(\nabla u)+\chi_{E} G(\nabla u)\right) \mathrm{d} x+P(E, \Omega),
$$

where $E \subset \Omega$ is a set of finite perimeter, $u \in W^{1, p}(\Omega), p>1, \chi_{E}$ is the characteristic function of the set $E$ and $P(E, \Omega)$ denotes the perimeter of $E$ in $\Omega$. We assume that $F, G: \mathbb{R}^{n} \rightarrow \mathbb{R}$ are $C^{1}$ integrands satisfying, for $p>1$ and positive constants $\ell, L, \alpha, \beta>0$ and $\mu \geq 0$, the following growth and uniform strong $p$-convexity hypotheses:

$$
\begin{gathered}
0 \leq F(\xi) \leq L\left(\mu^{2}+|\xi|^{2}\right)^{\frac{p}{2}} \\
\int_{\Omega} F(\xi+\nabla \varphi) \mathrm{d} x \geq \int_{\Omega}\left(F(\xi)+\ell\left(\mu^{2}+|\xi|^{2}+|\nabla \varphi|^{2}\right)^{\frac{p-2}{2}}|\nabla \varphi|^{2}\right) \mathrm{d} x
\end{gathered}
$$

\footnotetext{
Keywords and phrases. Regularity, nonlinear variational problem, free interfaces.

1 Dipartimento di Ingegneria - Università del Sannio, Corso Garibaldi 82100 Benevento, Italy. carozza@unisannio.it

2 Department of Mathematical Sciences, Carnegie Mellon University, PA 15213-3890 Pittsburgh, USA.

fonseca@andrew. cmu.edu

3 Università di Napoli "Federico II" Dipartimento di Mat. e Appl. 'R. Caccioppoli', Via Cintia, 80126 Napoli, Italy.

antpassa@unina.it
} 
and

$$
\begin{gathered}
0 \leq G(\xi) \leq \beta L\left(\mu^{2}+|\xi|^{2}\right)^{\frac{p}{2}} \\
\int_{\Omega} G(\xi+\nabla \varphi) \mathrm{d} x \geq \int_{\Omega}\left(G(\xi)+\alpha \ell\left(\mu^{2}+|\xi|^{2}+|\nabla \varphi|^{2}\right)^{\frac{p-2}{2}}|\nabla \varphi|^{2}\right) \mathrm{d} x
\end{gathered}
$$

for every $\xi \in \mathbb{R}^{n}$ and $\varphi \in C_{0}^{1}(\Omega)$.

We are interested in the following constrained problem

$$
\min \left\{\mathcal{I}(u, E): u=u_{0} \text { on } \partial \Omega,|E|=d\right\},
$$

where $u_{0} \in W^{1, p}(\Omega)$ and $0<d<|\Omega|$ are prescribed. Note that the strong convexity of $F$ and $G$, expressed by (F2) and (G2), by virtue of Theorem 1.4 below, ensures the existence of solutions of the problem (P).

Energies with surface terms competing with a volume term appear in a plethora of phenomena in materials science such as models for optimal design [4], phase transitions [18], liquid crystals [19], epitaxy [12] (see also [11]).

Our first regularity result is the following:

Theorem 1.1. Let $F$ and $G$ satisfy assumptions $(\mathrm{F} 1)-(\mathrm{F} 2)$ and $(\mathrm{G} 1)-(\mathrm{G} 2)$, respectively. Assume, in addition, that $F$ is p-homogeneous, i.e., $F(t \xi)=t^{p} F(\xi)$, for all $t \geq 0$. If $(u, E)$ is a minimizer of problem $(P)$, then $u \in C_{\mathrm{loc}}^{0, \frac{1}{p^{\prime}}}(\Omega)$, where $p^{\prime}$ denotes the Hölder's conjugate exponent of $p$, i.e., $p^{\prime}=\frac{p}{p-1}$. Moreover, $\mathcal{H}^{n-1}((\partial E \backslash$ $\left.\left.\partial^{*} E\right) \cap \Omega\right)=0$.

Previous results in this direction have been obtained in [4] and [20]. Precisely, Ambrosio and Buttazzo [4] and Lin [20] considered problems of the form

$$
\int_{\Omega}\left(\sigma_{E}(x)|\nabla u|^{2}\right) \mathrm{d} x+P(E, \Omega)
$$

with $u=0$ on $\partial \Omega$ and $\sigma_{E}(x):=a \chi_{E}+b \chi_{\Omega \backslash E}$ for $a$ and $b$ positive constants. It was proven in [4] that minimizers of (1.2) exist and that if $(u, E)$ is a minimal configuration then $u$ is locally Hölder continuous in $\Omega$ and, up to a set of $\mathcal{H}^{n-1}$ measure zero, there is no difference between the theoretic measure boundary of $E$ and its topological boundary. Recently, in [8], it has been proven that there exists $\gamma=\gamma(n)$ such that, for a minimal configuration $(u, E)$ of (1.2) if $1<a / b<\gamma(n)$, then $u$ is locally Hölder continuous in $\Omega$ and $\partial^{*} E$, the reduced boundary of $E$, is a $C^{1, \alpha}$-hypersurface. Moreover, Lin [20] showed that if $(u, E)$ is a minimizer of $(1.2)$ among all configurations such that $u$ and $\partial E$ are prescribed on $\partial \Omega$, then $u \in C^{0,1 / 2}(\Omega)$ and $\partial^{*} E$, the reduced boundary of $E$, is a $C^{1, \alpha}$-hypersurface away from a singular set $\Sigma$ of $\mathcal{H}^{n-1}$ measure zero. In [21], Lin and Kohn establish a partial regularity result for the boundary of the minimal set of the problem

$$
\mathcal{I}(u, E):=\int_{\Omega}\left(F(x, u, \nabla u)+\chi_{E} G(x, u, \nabla u)\right) \mathrm{d} x+P(E, \Omega),
$$

subject to the following constraints

$$
u=\Phi \text { on } \partial \Omega \text { and }|E|=d,
$$

requiring that $F$ and $G$ satisfy severe structure assumptions and have quadratic growth. A more detailed analysis of the minimal configurations of $(\mathrm{P})$ was carried out in the two dimensional case by Larsen in [19]. However, also in this case only partial regularity of $\partial^{*} E$ is obtained.

All minimum problems considered in the above mentioned papers have bulk energies of Dirichlet type with quadratic growth, i.e., of the form $|\cdot|^{2}$. Here, in Theorem 1.1 we treat constrained problems, we do not require any additional structure assumption on the bulk energies, and we assume $p$-growth (not necessarily $p=2$ ) with respect to the gradient. 
We point out that the Hölder exponent $\frac{1}{p^{\prime}}$ in Theorem 1.1 is critical, in the sense that the two terms in the energy functional (1.1) locally have the same dimension $n-1$ (under appropriate scalings). Actually, we will show that $u \in C_{\mathrm{loc}}^{0, \frac{1}{p^{\prime}}+\delta}(\Omega)$, for some $\delta>0$, under suitable conditions on the eigenvalues of $F$ and $G$, that, together with a result in [23] (see Thm. 2.3 in Sect. 2), allows us to conclude that $\partial^{*} E$ is a $C^{1, \tilde{\delta}}$ hypersurface, for some $0<\widetilde{\delta}<1$. More precisely, we have

Theorem 1.2. Let $F$ and $G$ satisfy assumptions (F1)-(F2) and (G1)-(G2), respectively. There exist $\gamma=$ $\gamma\left(n, p, \frac{\ell}{L}\right)<1$ and $\widetilde{\sigma}=\widetilde{\sigma}(n, p)>0$ such that if

$$
\left(\frac{\beta}{\alpha+1}\right)\left(\frac{\beta+1}{\alpha+1}\right)^{\tilde{\sigma}} \leq \gamma
$$

and if $(u, E)$ is a minimizer of problem $(P)$, then $u \in C_{\mathrm{loc}}^{0, \frac{1}{p^{\prime}}+\delta}(\Omega)$ for some positive $\delta$ depending on $n, p, \alpha, \beta$. Moreover $\partial^{*} E$ is a $C^{1, \widetilde{\delta}}$-hypersurface in $\Omega$, for some $\widetilde{\delta}<\frac{1}{2}$ depending on $n, p, \alpha, \beta$, and $\mathcal{H}^{s}\left(\left(\partial E \backslash \partial^{*} E\right) \cap \Omega\right)=0$ for all $s>n-8$.

Note that in Theorem 1.2 we do not impose the $p$-homogeneity of $F$, condition that we required in Theorem 1.1 only to avoid heavy technicalities.

Consider the prototype integrands

$$
F(\xi):=L\left(\mu^{2}+|\xi|^{2}\right)^{\frac{p}{2}} \quad \text { and } \quad G(\xi):=\beta L\left(\mu^{2}+|\xi|^{2}\right)^{\frac{p}{2}} .
$$

In this case the parameter $\alpha$ in assumption (G2) coincides with $\beta$ in assumption (G1), and condition (1.4) reduces to

$$
\beta \leq \frac{\gamma}{1-\gamma},
$$

with $\gamma=\gamma(n, p)<1$.

The functional (1.2) is a particular case of (1.1), setting

$$
F(\xi):=b|\xi|^{2} \quad \text { and } \quad G(\xi):=(a-b)|\xi|^{2}, \quad a>b .
$$

In this case, the parameters $\alpha, \beta$ in (G2) and (G1) are given by

$$
\beta=\alpha:=\frac{a-b}{b}
$$

and condition (1.4) becomes

$$
1<\frac{a}{b} \leq \frac{1}{1-\gamma}
$$

So, Theorem 1.2 gives back Theorem 2 in [8] as a particular case.

Further, without imposing any condition on the eigenvalues of the integrands, we are still able to obtain the following partial regularity result:

Theorem 1.3. Assume that (F1)-(F2) and (G1)-(G2) hold and let $(u, E)$ be a minimizer of problem $(P)$. Then there exists an open set $\Omega_{0} \subset \Omega$ with full measure such that $u \in C^{0, \eta}\left(\Omega_{0}\right)$, for every positive $\eta<1$. In addition, $\partial^{*} E \cap \Omega_{0}$ is a $C^{1, \tilde{\eta}}$-hypersurface in $\Omega_{0}$, for every $0<\widetilde{\eta}<\frac{1}{2}$, and $\mathcal{H}^{s}\left(\left(\partial E \backslash \partial^{*} E\right) \cap \Omega_{0}\right)=0$ for all $s>n-8$.

In the study of regularity properties, the constraint $|E|=d$ introduces extra difficulties, since one can work only with variations which keep the volume constant. The next theorem allows us to circumvent this extra difficulties, ensuring that every minimizer of the constrained problem $(\mathrm{P})$ is also a minimizer of a suitable unconstrained energy functional with a volume penalization. 
Theorem 1.4. There exists $\lambda_{0}>0$ such that if $(u, E)$ is a minimizer of the functional

$$
\mathcal{I}_{\lambda}(v, A):=\int_{\Omega}\left(F(\nabla v)+\chi_{A} G(\nabla v)\right) \mathrm{d} x+P(A, \Omega)+\lambda|| A|-d|
$$

for some $\lambda \geq \lambda_{0}$ and among all configurations $(v, A)$ such that $v=u_{0}$ on $\partial \Omega$, then $|E|=d$ and $(u, E)$ is a minimizer of problem $(P)$. Conversely, if $(u, E)$ is a minimizer of the problem $(P)$, then it is a minimizer of (1.5), for all $\lambda \geq \lambda_{0}$.

Theorem 1.4 is a straightforward modification of a result due to Esposito and Fusco (see [8], Thm. 1). Since several modifications are needed, we present its proof in Section 3 for the reader's convenience. Similar arguments have been used in Fonseca, Fusco, Leoni and Millot [11] (see also Alt and Caffarelli [1]).

From the point of view of regularity, the extra term $\lambda|| A|-d|$ is a higher order, negligible perturbation, in the sense that if $x_{0} \in \partial^{*} E \cap \partial \Omega$ then $\left|E \cap B_{\varrho}\left(x_{0}\right)\right|$ decays as $\varrho^{n}$ as $\varrho \rightarrow 0^{+}$while the leading term $\int_{B_{\varrho}\left(x_{0}\right)}\left(F(\nabla u)+\chi_{E} G(\nabla u)\right) \mathrm{d} x+P\left(E, B_{\varrho}\left(x_{0}\right)\right)$ decays as $\varrho^{n-1}$.

The proof of Theorem 1.1 is based on a decay estimate for the gradient of the minimizer $u$, obtained by blowing-up the minimizer $u$ in small balls. We establish that the minimizers of the rescaled problems converge to a Hölder continuous function $v$, and we show that $u$ and $v$ are "close enough" (with respect to the norm in the Sobolev space $W^{1, p}$ ) in order to ensure that $u$ inherits the regularity estimates of $v$.

Theorems 1.2 and 1.3 are obtained by a comparison argument between the minimizer of $(\mathrm{P})$ and the minimizer of a suitable convex scalar functional with $p$-growth, for which regularity results are well known. Also here, we show that the two minimizers are "close" enough to share the same good regularity properties. We remark that in this comparison argument we need that $u$ is a real valued function. In fact, in the vectorial setting (see [22]) minimizers of regular variational functionals may have singularities and only partial regularity results are known (see for example $[3,7,13]$ ).

This paper is organized as follows: in Section 2 we fix the notation and collect standard preliminary results. The proof of Theorem 1.4 is given in Section 3, since the result is needed in the proofs of the other theorems. The proofs of Theorems 1.1, 1.2 and 1.3 are presented in Sections 4, 5 and 6, respectively.

\section{Notations AND PRELIMinaRY RESUlts}

In this paper we follow usual convention and denote by $c$ a general constant that may vary from expression to expression, even within the same line of estimates. Relevant dependencies on parameters and special constants will be suitably emphasized using parentheses or subscripts. The norm we use in $\mathbb{R}^{n}$ is the standard Euclidean norm, and it will be denoted by $|\cdot|$. In particular, for vectors $\xi, \eta \in \mathbb{R}^{n}$ we write $\langle\xi, \eta\rangle$ for the inner product of $\xi$ and $\eta$, and $|\xi|:=\langle\xi, \xi\rangle^{\frac{1}{2}}$ is the corresponding Euclidean norm. When $a, b \in \mathbb{R}^{n}$ we write $a \otimes b$ for the tensor product defined as the matrix that has the element $a_{r} b_{s}$ in its $r$ th row and $s$ th column. Observe that $(a \otimes b) x=(b \cdot x) a$ for $x \in \mathbb{R}^{n}$, and $|a \otimes b|=|a||b|$. When $F: \mathbb{R}^{n} \rightarrow \mathbb{R}$ is $C^{1}$, we write

$$
D_{\xi} F(\xi)[\eta]:=\left.\frac{\mathrm{d}}{\mathrm{d} t}\right|_{t=0} F(\xi+t \eta)
$$

if $\xi, \eta \in \mathbb{R}^{n}$. It is convenient to express the convexity and growth conditions of the integrands in terms of an auxiliary function defined for all $\xi \in \mathbb{R}^{n}$ as

$$
V(\xi)=V_{p, \mu}(\xi):=\left(\mu^{2}+|\xi|^{2}\right)^{\frac{p-2}{4}} \xi,
$$

where $\mu \geq 0$ and $p \geq 1$. We recall the following lemmas.

Lemma 2.1. Let $1<p<\infty$ and $0 \leq \mu \leq 1$. There exists a constant $c=c(n, N, p)>0$ such that

$$
c^{-1}\left(\mu^{2}+|\xi|^{2}+|\eta|^{2}\right)^{\frac{p-2}{2}} \leq \frac{\left|V_{p, \mu}(\xi)-V_{p, \mu}(\eta)\right|^{2}}{|\xi-\eta|^{2}} \leq c\left(\mu^{2}+|\xi|^{2}+|\eta|^{2}\right)^{\frac{p-2}{2}}
$$

for all $\xi, \eta \in \mathbb{R}^{n}$. 
For the proof we refer to [17], Lemma 8.3. The next lemma can be found in ([15], Lem. 2.1) and ([2], Lem. 2.1) for $p \geq 2$ and $1<p<2$, respectively.

Lemma 2.2. For $1<p<\infty$ and all $\xi, \eta \in \mathbb{R}^{n}$ one has

$$
\frac{1}{c} \leq \frac{\left.\left.\int_{0}^{1}\left(\mu^{2}+\mid \xi+t \eta\right)\right|^{2}\right)^{\frac{p-2}{2}} \mathrm{~d} t}{\left(\mu^{2}+|\xi|^{2}+|\eta|^{2}\right)^{\frac{p-2}{2}}} \leq c,
$$

where $c$ depends only on $p$.

It is well-known that for convex $C^{1}$ integrands, the assumptions (F1) and (G1) yield the upper bounds

$$
\left|D_{\xi} F(\xi)\right| \leq c_{1}\left(\mu^{2}+|\xi|^{2}\right)^{\frac{p-1}{2}} \quad\left|D_{\xi} G(\xi)\right| \leq c_{2}\left(\mu^{2}+|\xi|^{2}\right)^{\frac{p-1}{2}}
$$

for all $\xi \in \mathbb{R}^{n}$, where we can use $c_{1}:=2^{p} L$ and $c_{2}:=2^{p} \beta L$ (see [17]).

Also, if $F$ and $G$ satisfy (F2) and (G2), respectively, then the following strong $p$-monotonicity conditions hold:

$$
\begin{aligned}
& \left\langle D_{\xi} F(\xi)-D_{\xi} F(\eta), \xi-\eta\right\rangle \geq c(p) \ell|V(\xi)-V(\eta)|^{2} \\
& \left\langle D_{\xi} G(\xi)-D_{\xi} G(\eta), \xi-\eta\right\rangle \geq c(p) \alpha \ell|V(\xi)-V(\eta)|^{2}
\end{aligned}
$$

for all $\xi, \eta \in \mathbb{R}^{n}$ and some $c(p)>0$. In fact, (F2) and (G2) are equivalent to the convexity of the functions

$$
\xi \rightarrow \widetilde{F}(\xi):=F(\xi)-\ell\left(\mu^{2}+|\xi|^{2}\right)^{\frac{p}{2}}
$$

and

$$
\xi \rightarrow \widetilde{G}(\xi):=G(\xi)-\alpha \ell\left(\mu^{2}+|\xi|^{2}\right)^{\frac{p}{2}},
$$

respectively (see for example [17], p. 164). Hence, the convexity of $\widetilde{F}$ implies

$$
F(\xi)-\ell\left(\mu^{2}+|\xi|^{2}\right)^{\frac{p}{2}} \geq F(\eta)-\ell\left(\mu^{2}+|\eta|^{2}\right)^{\frac{p}{2}}+\left\langle D_{\xi} F(\eta), \xi-\eta\right\rangle-\ell p\left\langle\left(\mu^{2}+|\eta|^{2}\right)^{\frac{p}{2}-1} \eta, \xi-\eta\right\rangle
$$

and

$$
F(\eta)-\ell\left(\mu^{2}+|\eta|^{2}\right)^{\frac{p}{2}} \geq F(\xi)-\ell\left(\mu^{2}+|\xi|^{2}\right)^{\frac{p}{2}}+\left\langle D_{\xi} F(\xi), \eta-\xi\right\rangle-\ell p\left\langle\left(\mu^{2}+|\xi|^{2}\right)^{\frac{p}{2}-1} \xi, \eta-\xi\right\rangle .
$$

Summing previous inequalities and using Lemmas 2.2 and 2.1, we obtain

$$
\begin{aligned}
\left\langle D_{\xi} F(\xi)-D_{\xi} F(\eta), \xi-\eta\right\rangle & \geq \ell p\left\langle\left(\mu^{2}+|\xi|^{2}\right)^{\frac{p}{2}-1} \xi, \xi-\eta\right\rangle-\ell p\left\langle\left(\mu^{2}+|\eta|^{2}\right)^{\frac{p}{2}-1} \eta, \xi-\eta\right\rangle \\
& \geq \ell p\left\langle\left(\mu^{2}+|\xi|^{2}\right)^{\frac{p}{2}-1} \xi-\left(\mu^{2}+|\eta|^{2}\right)^{\frac{p}{2}-1} \eta, \xi-\eta\right\rangle \\
& \geq c(p) \ell \int_{0}^{1}\left(\mu^{2}+|\xi+t(\eta-\xi)|^{2}\right)^{\frac{p}{2}-1} \mathrm{~d} t|\xi-\eta|^{2} \\
& \geq c(p) \ell|V(\xi)-V(\eta)|^{2},
\end{aligned}
$$

i.e., the first inequality in (2.3). The second inequality in (2.3) can be derived arguing similarly.

Further, if $F$ and $G$ are $C^{2}$, then (F2) and (G2) are equivalent to the following standard strong $p$-ellipticity conditions

$$
\left\langle D^{2} F(\xi) \eta, \eta\right\rangle \geq c_{3}\left(\mu^{2}+|\xi|^{2}\right)^{\frac{p-2}{2}}|\eta|^{2}, \quad\left\langle D^{2} G(\xi) \eta, \eta\right\rangle \geq c_{4}\left(\mu^{2}+|\xi|^{2}\right)^{\frac{p-2}{2}}|\eta|^{2}
$$

for all $\xi, \eta \in \mathbb{R}^{n}$, where $c_{i}$ are positive constants of form $c_{3}=c(p) \ell$ and $c_{4}=c(p) \alpha \ell$, respectively.

The next lemma establishes that the uniform strong $p$-convexity assumptions (F2) and (G2) yield growth conditions from below for the functions $F$ and $G$. 
Lemma 2.3. Suppose that $H: \mathbb{R}^{n} \rightarrow[0,+\infty)$ is a $C^{1}$ function such that

$$
0 \leq H(\xi) \leq \widetilde{L}\left(\mu^{2}+|\xi|^{2}\right)^{\frac{p}{2}}
$$

for all $\xi \in \mathbb{R}^{n}$, where $p>1,0 \leq \mu \leq 1, \widetilde{L}>0$. Assume, in addition, that

$$
\int_{Q} H(\xi+\nabla \varphi) \mathrm{d} x \geq \int_{Q} H(\xi)+\widetilde{\ell}\left(\mu^{2}+|\xi|^{2}+|\nabla \varphi|^{2}\right)^{\frac{p-2}{2}}|\nabla \varphi|^{2} \mathrm{~d} x
$$

for all $\xi \in \mathbb{R}^{n}, \varphi \in C_{0}^{1}(Q), Q \subset \mathbb{R}^{n}$ and for some positive constant $\tilde{\ell}$. Then there exists a positive constant $c(p, \widetilde{L}, \tilde{\ell}, \mu)$ such that

$$
H(\xi) \geq \frac{\tilde{\ell}}{2}\left(\mu^{2}+|\xi|^{2}\right)^{\frac{p}{2}}-c(p, \widetilde{L}, \widetilde{\ell}, \mu) \quad \text { for all } \xi \in \mathbb{R}^{n} .
$$

Proof. We use again the fact that assumption (2.5) is equivalent to the convexity of the function

$$
\xi \rightarrow K(\xi):=H(\xi)-\widetilde{\ell}\left(\mu^{2}+|\xi|^{2}\right)^{\frac{p}{2}} .
$$

Hence

$$
K(\xi) \geq K(0)+\left\langle D_{\xi} K(0), \xi\right\rangle
$$

or, equivalently,

$$
H(\xi) \geq \widetilde{\ell}\left(\mu^{2}+|\xi|^{2}\right)^{\frac{p}{2}}+H(0)-\widetilde{\ell} \mu^{p}+\left\langle D_{\xi} H(0), \xi\right\rangle
$$

for all $\xi \in \mathbb{R}^{n}$. By (2.4) and (2.2), we have that

$$
H(0) \geq 0 \quad \text { and } \quad\left|D_{\xi} H(0)\right| \leq 2^{p} \widetilde{L} \mu^{p-1},
$$

and by Young's inequality

$$
\begin{aligned}
\left|\left\langle D_{\xi} H(0), \xi\right\rangle\right| & =\left|\left\langle(\widetilde{\ell})^{-\frac{1}{p}} D_{\xi} H(0),(\widetilde{\ell})^{\frac{1}{p}} \xi\right\rangle\right| \leq c(\varepsilon)(\widetilde{\ell})^{-\frac{1}{p-1}}\left|D_{\xi} H(0)\right|^{\frac{p}{p-1}}+\varepsilon \widetilde{\ell}|\xi|^{p} \\
& \leq c(\varepsilon) 2^{\frac{p^{2}}{p-1}}(\widetilde{\ell})^{-\frac{1}{p-1}} \widetilde{L}^{\frac{p}{p-1}} \mu^{p}+\varepsilon \widetilde{\ell}\left(\mu^{2}+|\xi|^{2}\right)^{\frac{p}{2}} .
\end{aligned}
$$

Inserting (2.8) in (2.7), we get

$$
H(\xi) \geq \widetilde{\ell}\left(\mu^{2}+|\xi|^{2}\right)^{\frac{p}{2}}-c(\varepsilon) 2^{\frac{p^{2}}{p-1}}(\widetilde{\ell})^{-\frac{1}{p-1}} \widetilde{L}^{\frac{p}{p-1}} \mu^{p}-\varepsilon \widetilde{\ell}\left(\mu^{2}+|\xi|^{2}\right)^{\frac{p}{2}}-\widetilde{\ell} \mu^{p}
$$

and, choosing $\varepsilon=\frac{1}{2}$, we conclude that

$$
H(\xi) \geq \frac{\tilde{\ell}}{2}\left(\mu^{2}+|\xi|^{2}\right)^{\frac{p}{2}}-c_{p}\left(\frac{\widetilde{L}^{p}}{\widetilde{\ell}}\right)^{\frac{1}{p-1}} \mu^{p}-\widetilde{\ell} \mu^{p} .
$$

As already mentioned in the Introduction, we will compare the minimizer $u$ of the problem $(\mathrm{P})$ with the minimizer of a suitable regular convex variational integral. In order to take advantage of the comparison argument, we will need the following regularity result (see [10], Thm. 2.2)

Theorem 2.1. Let $H: \mathbb{R}^{n} \rightarrow[0,+\infty)$ be a continuous function such that

$$
0 \leq H(\xi) \leq \widetilde{L}\left(\mu^{2}+|\xi|^{2}\right)^{\frac{p}{2}}
$$


for all $\xi \in \mathbb{R}^{n}$, where $p>1,0 \leq \mu \leq 1, \widetilde{L}>0$. Suppose, in addition, that

$$
\int_{Q} H(\xi+\nabla \varphi) \mathrm{d} x \geq \int_{Q} H(\xi)+\widetilde{\ell}\left(\mu^{2}+|\xi|^{2}+|\nabla \varphi|^{2}\right)^{\frac{p-2}{2}}|\nabla \varphi|^{2} \mathrm{~d} x
$$

for all $\xi \in \mathbb{R}^{n}, \varphi \in C_{0}^{1}(Q), Q \subset \mathbb{R}^{n}$ and for some positive constant $\tilde{\ell}$. If $v \in W^{1, p}(\Omega)$ is a local minimizer of the functional

$$
\mathcal{H}(w, \Omega):=\int_{\Omega} H(\nabla w) \mathrm{d} x
$$

i.e.,

$$
\mathcal{H}\left(v, B_{r}\left(x_{0}\right)\right)=\min \left\{\mathcal{H}\left(w, B_{r}\left(x_{0}\right)\right): w \in v+W_{0}^{1, p}\left(B_{r}\left(x_{0}\right)\right)\right\} \quad \text { for all } B_{r}\left(x_{0}\right) \subset \Omega,
$$

then $v$ is locally Lipschitz in $\Omega$, and

$$
\operatorname{ess} \sup _{B_{\frac{R}{2}}\left(x_{0}\right)}\left(\mu^{2}+|\nabla v|^{2}\right)^{\frac{p}{2}} \leq c(n, \widetilde{L}, \tilde{\ell}, p) f_{B_{R}\left(x_{0}\right)}\left(\mu^{2}+|\nabla v|^{2}\right)^{\frac{p}{2}} \mathrm{~d} x
$$

for every $B_{\frac{R}{2}}\left(x_{0}\right) \subset B_{R}\left(x_{0}\right) \subset \Omega$.

In what follows, we will need a more explicit dependence on the eigenvalues of $H$ of the constant in (2.9). Actually, a careful inspection of the proof of Theorem 2.2 in [10] reveals that the constant in estimate (2.9) is of the type

$$
c(n, \widetilde{L}, \widetilde{\ell}, p)=c\left(\frac{\widetilde{L}}{\widetilde{\ell}}\right)^{\frac{2 n}{p}}
$$

where $c=c(n, p) \geq 1$.

The following is a technical iteration lemma (see [17], Lem. 7.3)

Lemma 2.4. Let $\varphi$ be a nonnegative, nondecreasing function and assume that there exist $\vartheta \in(0,1), \bar{R}>0$, and $0<\beta<\gamma$ such that

$$
\varphi(\vartheta r) \leq \vartheta^{\gamma} \varphi(r)+b r^{\beta}
$$

for all $0<r \leq \bar{R}$. Then we have

$$
\varphi(\rho) \leq C\left\{\left(\frac{\rho}{r}\right)^{\beta} \varphi(r)+b \rho^{\beta}\right\},
$$

for every $0<\rho<r \leq \bar{R}$, with $C=C(\vartheta, \beta, \gamma)$.

The next result relates the decay estimate for the gradient of a Sobolev function with its Hölder regularity properties (see [14], Thm. 1.1, p. 64 and [16], Thm. 7.19)

Theorem 2.2 (Morrey's Lemma). Let $u \in W^{1,1}(\Omega)$ and suppose that there exist positive constants $K$, $0<\alpha \leq 1$ such that

$$
\int_{B_{r}(x)}|\nabla u| \mathrm{d} x \leq K r^{n-1+\alpha},
$$

for all balls $B(x, r) \subset \Omega, x \in \Omega, r>0$. Then $u \in C^{0, \alpha}(\Omega)$.

Given a Borel set $E$ in $\mathbb{R}^{n}, P(E, \Omega)$ denotes the perimeter of $E$ in $\Omega$, defined as

$$
P(E, \Omega)=\sup \left\{\int_{E} \operatorname{div} \phi \mathrm{d} x: \quad \phi \in C_{0}^{1}\left(\Omega ; \mathbb{R}^{n}\right),|\phi| \leq 1\right\} .
$$


It is known that, for a set of finite perimeter $E$, one has

$$
P(E, \Omega)=\mathcal{H}^{n-1}\left(\partial^{*} E\right)
$$

where

$$
\partial^{*} E=\left\{x \in \Omega: \limsup _{\rho \rightarrow 0^{+}} \frac{P\left(E, B_{\rho}(x)\right)}{\rho^{n-1}}>0\right\}
$$

is the reduced boundary of $E$ (for more details we refer to [5]).

Given a set $E \subset \Omega$ of finite perimeter in $\Omega$, for every ball $B_{r}(x) \Subset \Omega$ we measure how far $E$ is from being an area minimizer in the ball by setting

$$
\psi\left(E, B_{r}(x)\right):=P\left(E, B_{r}(x)\right)-\min \left\{P\left(A, B_{r}(x)\right): A \Delta E \Subset B_{r}(x), \chi_{A} \in B V\left(\mathbb{R}^{n}\right)\right\} .
$$

The following regularity result, due to Tamanini (see [23]), asserts that if the excess $\psi\left(E, B_{r}(x)\right)$ decays fast enough when $r \rightarrow 0$, then $E$ has essentially the same regularity properties of an area minimizing set.

Theorem 2.3. Let $\Omega$ be an open subset of $\mathbb{R}^{n}$ and let $E$ be a set of finite perimeter satisfying, for some $\delta \in\left(0, \frac{1}{2}\right)$,

$$
\psi\left(E, B_{r}(x)\right) \leq c r^{n-1+\delta}
$$

for every $x \in \Omega$ and every $r \in\left(0, r_{0}\right)$, with $c=c(x), r_{0}=r_{0}(x)$ local positive constants. Then $\partial^{*} E$ is a $C^{1, \delta}$-hypersurface in $\Omega$ and $\left.\mathcal{H}^{s}\left(\left(\partial E \backslash \partial^{*} E\right) \cap \Omega\right)\right)=0$ for all $s>n-8$.

\section{Proof of Theorem 1.4}

This section is devoted to the proof of Theorem 1.4, which follows closely that of Theorem 1 in [8]. Since several modifications are needed, we present it here for the convenience of the reader.

Proof of Theorem 1.4.

Step 1. We prove the first part of Theorem 1.4 arguing by contradiction. Assume that there exist a sequence $\left\{\lambda_{h}\right\}_{h \in \mathbb{N}}$ such that $\lambda_{h} \rightarrow \infty$ as $h \rightarrow \infty$, and a sequence of configurations $\left\{\left(u_{h}, E_{h}\right)\right\}$ minimizing $\mathcal{I}_{\lambda_{h}}$ such that $u_{h}=u_{0}$ on $\partial \Omega$ and $\left|E_{h}\right| \neq d$ for all $h$. Notice that

$$
\mathcal{I}_{\lambda_{h}}\left(u_{h}, E_{h}\right) \leq \mathcal{I}\left(u_{0}, E_{0}\right)=: \Theta,
$$

where $E_{0} \subset \Omega$ is a fixed set of finite perimeter such that $\left|E_{0}\right|=d$. Assume that $\left|E_{h}\right|<d$ for a (not relabeled) subsequence (if $\left|E_{h}\right|>d$ the proof is similar). We claim that, for $h$ sufficiently large, there exists a configuration $\left(\tilde{u}_{h}, \widetilde{E}_{h}\right)$ such that $\mathcal{I}_{\lambda_{h}}\left(\tilde{u}_{h}, \widetilde{E}_{h}\right)<\mathcal{I}_{\lambda_{h}}\left(u_{h}, E_{h}\right)$, thus proving that $\left|E_{h}\right|=d$ for all $h$ sufficiently large, say $\lambda \geq \lambda_{0}$.

By our assumptions on $F$ and $G$, it follows that the sequence $\left\{u_{h}\right\}$ is bounded in $W^{1, p}(\Omega)$, the perimeters of the sets $E_{h}$ are bounded, and $\left|E_{h}\right| \rightarrow d$. Therefore, without loss of generality, we may assume, possibly extracting a subsequence (not relabeled), that there exists a configuration $(u, E)$ such that $u_{h} \rightarrow u$ weakly in $W^{1, p}(\Omega), \chi_{E_{h}} \rightarrow \chi_{E}$ a.e. in $\Omega$, and $E$ is a set of finite perimeter in $\Omega$ with $|E|=d$.

Step 2. Construction of $\left(\tilde{u}_{h}, \widetilde{E}_{h}\right)$. Fix a point $x \in \partial^{*} E \cap \Omega$ (such a point exists since $E$ has finite perimeter in $\Omega, 0<|E|<|\Omega|$, and $\Omega$ is connected). By De Giorgi's structure theorem for sets of finite perimeter (see [5], Thm. 3.59), the sets $E_{r}=(E-x) / r$ converge locally in measure to the half space $H=\left\{z \cdot \nu_{E}(x)>0\right\}$, i.e., $\chi_{E_{r}} \rightarrow \chi_{H}$ in $L_{\mathrm{loc}}^{1}\left(\mathbb{R}^{n}\right)$, where $\nu_{E}(x)$ is the generalized inner normal to $E$ at $x$ (see [5], Def. 3.54). Let $y \in B_{1}(0) \backslash H$ be the point $y:=-\nu_{E}(x) / 2$. Given $\varepsilon>0$ and small (to be chosen at the end of the proof), since $\chi_{E_{r}} \rightarrow \chi_{H}$ in $L^{1}\left(B_{1}(0)\right)$ there exists $r>0$ such that

$$
B_{2 r}(x) \subset \Omega, \quad\left|E_{r} \cap B_{1 / 2}(y)\right|<\varepsilon, \quad\left|E_{r} \cap B_{1}(y)\right| \geq\left|E_{r} \cap B_{1 / 2}(0)\right|>\frac{\omega_{n}}{2^{n+2}},
$$


where $\omega_{n}$ denotes the measure of the unit ball of $\mathbb{R}^{n}$. Therefore, setting $x_{r}:=x+r y \in \Omega$, we have

$$
B_{r}\left(x_{r}\right) \subset \Omega, \quad\left|E \cap B_{r / 2}\left(x_{r}\right)\right|<\varepsilon r^{n}, \quad\left|E \cap B_{r}\left(x_{r}\right)\right|>\frac{\omega_{n} r^{n}}{2^{n+2}} .
$$

Assume, without loss of generality, that $x_{r}=0$, and in the sequel denote the open ball centered at the origin and with radius $r>0$ by $B_{r}$. From the convergence of $\left\{E_{h}\right\}$ to $E$ we have that, for all $h$ sufficiently large,

$$
\left|E_{h} \cap B_{r / 2}\right|<\varepsilon r^{n}, \quad\left|E_{h} \cap B_{r}\right|>\frac{\omega_{n} r^{n}}{2^{n+2}} .
$$

Define the bi-Lipschitz map $\phi: B_{r} \rightarrow B_{r}$ by

$$
\Phi(x):= \begin{cases}\left(1-\sigma\left(2^{n}-1\right)\right) x & \text { if }|x|<\frac{r}{2}, \\ x+\sigma\left(1-\frac{r^{n}}{|x|^{n}}\right) x & \text { if } \frac{r}{2} \leq|x|<r, \\ x & \text { if }|x| \geq r\end{cases}
$$

for some fixed $0<\sigma<1 / 2^{n}$, to be determined later, such that, setting

$$
\widetilde{E}_{h}:=\Phi\left(E_{h}\right), \quad \tilde{u}_{h}:=u_{h} \circ \Phi^{-1},
$$

we have $\left|\widetilde{E}_{h}\right|<d$. We obtain

$$
\begin{aligned}
\mathcal{I}_{\lambda_{h}}\left(u_{h}, E_{h}\right)-\mathcal{I}_{\lambda_{h}}\left(\tilde{u}_{h}, \widetilde{E}_{h}\right)= & {\left[\int_{B_{r}}\left(F\left(\nabla u_{h}\right)+\chi_{E_{h}} G\left(\nabla u_{h}\right)\right) \mathrm{d} x-\int_{B_{r}}\left(F\left(\nabla \tilde{u}_{h}\right)+\chi_{\widetilde{E}_{h}} G\left(\nabla \tilde{u}_{h}\right)\right) \mathrm{d} y\right] } \\
& +\left(P\left(E_{h}, \bar{B}_{r}\right)-P\left(\widetilde{E}_{h}, \bar{B}_{r}\right)\right)+\lambda_{h}\left(\left|\widetilde{E}_{h}\right|-\left|E_{h}\right|\right) \\
= & I_{1, h}+I_{2, h}+I_{3, h} .
\end{aligned}
$$

Step 3. Estimate of $I_{1, h}$. We start by evaluating the gradient and the Jacobian determinant of $\Phi$ in the annulus $B_{r} \backslash B_{r / 2}$. If $r / 2<|x|<r$, then we have

$$
\frac{\partial \Phi_{i}}{\partial x_{j}}(x)=\left(1+\sigma-\frac{\sigma r^{n}}{|x|^{n}}\right) \delta_{i j}+n \sigma r^{n} \frac{x_{i} x_{j}}{|x|^{n+2}} \quad \text { for all } i, j=1, \ldots n
$$

and thus, if $\eta \in \mathbb{R}^{n}$,

$$
(\nabla \Phi \circ \eta) \cdot \eta=\left(1+\sigma-\frac{\sigma r^{n}}{|x|^{n}}\right)|\eta|^{2}+n \sigma r^{n} \frac{(x \cdot \eta)^{2}}{|x|^{n+2}}
$$

from which it follows that

$$
|\nabla \Phi \circ \eta| \geq\left(1+\sigma-\frac{\sigma r^{n}}{|x|^{n}}\right)|\eta| .
$$

From this inequality we easily deduce an estimate on the norm of $\nabla \Phi^{-1}$, precisely,

$$
\begin{aligned}
\left\|\nabla \Phi^{-1}(\Phi(x))\right\| & =\max _{|\eta|=1}\left|\nabla \Phi^{-1} \circ\left(\frac{\nabla \Phi \circ \eta}{|\nabla \Phi \circ \eta|}\right)\right|=\max _{|\eta|=1} \frac{1}{|\nabla \Phi \circ \eta|} \\
& \leq\left(1+\sigma-\frac{\sigma r^{n}}{|x|^{n}}\right)^{-1} \leq\left(1-\left(2^{n}-1\right) \sigma\right)^{-1} \quad \text { for all } x \in B_{r} \backslash B_{r / 2} .
\end{aligned}
$$

Concerning the Jacobian, we write, for $x \in B_{r} \backslash B_{r / 2}$,

$$
\Phi(x)=\varphi(|x|) \frac{x}{|x|}
$$


where

$$
\varphi(t)=t\left(1+\sigma-\frac{\sigma r^{n}}{t^{n}}\right), \quad \text { for all } t \in[r / 2, r] .
$$

Let $I$ denote the identity map in $\mathbb{R}^{n}$. Recalling that if $A=I+a \otimes b$ for some vectors $a, b \in \mathbb{R}^{n}$, then $\operatorname{det} A=1+a \cdot b$, a straightforward calculation gives for all $x \in B_{r} \backslash B_{r / 2}$

$$
J \Phi(x)=\varphi^{\prime}(|x|)\left(\frac{\varphi(|x|)}{|x|}\right)^{n-1}=\left(1+\sigma+\frac{(n-1) \sigma r^{n}}{|x|^{n}}\right)\left(1+\sigma-\frac{\sigma r^{n}}{|x|^{n}}\right)^{n-1} .
$$

We have

$$
\begin{aligned}
\left(1+\sigma-\frac{\sigma r^{n}}{|x|^{n}}\right)^{n-1} & =(1+\sigma)^{n-1}\left(1-\frac{\frac{\sigma r^{n}}{|x|^{n}}}{1+\sigma}\right)^{n-1} \geq(1+\sigma)^{n-1}\left(1-(n-1) \frac{\frac{\sigma r^{n}}{|x|^{n}}}{1+\sigma}\right) \\
& =(1+\sigma)^{n-2}\left(1+\sigma-(n-1) \frac{\sigma r^{n}}{|x|^{n}}\right) \geq 1+\sigma-(n-1) \frac{\sigma r^{n}}{|x|^{n}} .
\end{aligned}
$$

Since $x \in B_{r} \backslash B_{\frac{r}{2}}$, by (3.7) and (3.8) we have

$$
\begin{aligned}
J \Phi(x) & \geq\left(1+\sigma+(n-1) \frac{\sigma r^{n}}{|x|^{n}}\right)\left(1+\sigma-(n-1) \frac{\sigma r^{n}}{|x|^{n}}\right)=(1+\sigma)^{2}-(n-1)^{2} \frac{\sigma^{2} r^{2 n}}{|x|^{2 n}} \\
& \geq(1+\sigma)^{2}-4^{n}(n-1)^{2} \sigma^{2}=1+2 \sigma-\left(4^{n}(n-1)^{2}-1\right) \sigma^{2}>1+\sigma
\end{aligned}
$$

provided that we chose

$$
\sigma<\frac{1}{4^{n}(n-1)^{2}-1}
$$

On the other hand, from (3.7) we get also

$$
J \Phi(x) \leq 1+2^{n} n \sigma .
$$

Let us now turn to the estimate of $I_{1, h}$. Performing the change of variable $y=\Phi(x)$ in the second integral defining $I_{1, h}$, and observing that $\chi_{\widetilde{E}_{h}}(\Phi(x))=\chi_{E_{h}}(x)$, we get

$$
\begin{aligned}
I_{1, h}= & \int_{B_{r}}\left[F\left(\nabla u_{h}(x)\right)-F\left(\nabla u_{h}(x) \circ \nabla \Phi^{-1}(\Phi(x))\right) J \Phi(x)\right. \\
& +\chi_{E_{h}}(x)\left[G\left(\nabla u_{h}(x)\right)-G\left(\nabla u_{h}(x) \circ \nabla \Phi^{-1}(\Phi(x))\right) J \Phi(x)\right] \mathrm{d} x \\
= & : A_{1, h}+A_{2, h},
\end{aligned}
$$

where $A_{1, h}$ stands for the above integral evaluated in $B_{r / 2}$ and $A_{2, h}$ for the same integral evaluated in $B_{r} \backslash B_{r / 2}$. Recalling the definition of $\Phi$ in (3.3) and the growth assumptions on $F, G$ in (F1) and (G1), respectively, we get

$$
\begin{aligned}
A_{1, h}= & \int_{B_{r / 2}}\left[F\left(\nabla u_{h}(x)\right)-F\left(\nabla u_{h}(x) \circ\left(1-\sigma\left(2^{n}-1\right)\right)^{-1} I\right)\left(1-\sigma\left(2^{n}-1\right)\right)^{n}\right] \mathrm{d} x \\
& +\int_{B_{r / 2}} \chi_{E_{h}}(x)\left[G\left(\nabla u_{h}(x)\right)-G\left(\nabla u_{h}(x) \circ\left(1-\sigma\left(2^{n}-1\right)\right)^{-1} I\right)\left(1-\sigma\left(2^{n}-1\right)\right)^{n}\right] \mathrm{d} x \\
\geq & -\int_{B_{r / 2}} F\left(\nabla u_{h}(x) \circ\left(1-\sigma\left(2^{n}-1\right)\right)^{-1} I\right)\left(1-\sigma\left(2^{n}-1\right)\right)^{n} \mathrm{~d} x
\end{aligned}
$$




$$
\begin{aligned}
& -\int_{B_{r / 2}} \chi_{E_{h}}(x) G\left(\nabla u_{h}(x) \circ\left(1-\sigma\left(2^{n}-1\right)\right)^{-1} I\right)\left(1-\sigma\left(2^{n}-1\right)\right)^{n} \mathrm{~d} x \\
\geq & -c(p, \beta, L) \int_{B_{r / 2}}\left(1+\chi_{E_{h}}(x)\right)\left|\nabla u_{h}(x) \circ\left(1-\sigma\left(2^{n}-1\right)\right)^{-1} I\right|^{p}\left(1-\sigma\left(2^{n}-1\right)\right)^{n} \mathrm{~d} x \\
& -c(p, \beta, L) \mu^{p}\left(1-\sigma\left(2^{n}-1\right)\right)^{n} r^{n} \\
\geq & -c(p, \beta, L) \int_{B_{r / 2}}\left(1+\chi_{E_{h}}(x)\right)\left|\nabla u_{h}(x)\right|^{p}\left(1-\sigma\left(2^{n}-1\right)\right)^{n-p} \mathrm{~d} x \\
& -c(p, \beta, L) \mu^{p}\left(1-\sigma\left(2^{n}-1\right)\right)^{n} r^{n} \\
= & -c\left(1-\sigma\left(2^{n}-1\right)\right)^{n-p} \int_{B_{r / 2}}\left|\nabla u_{h}(x)\right|^{p} \mathrm{~d} x-c \mu^{p}\left(1-\sigma\left(2^{n}-1\right)\right)^{n} r^{n} \\
\geq & -C(n, p, \beta, L, \sigma, \mu)\left(\Theta+r^{n}\right)
\end{aligned}
$$

where we used (3.1). Recalling (3.5), (3.10) and (3.1) we have

$$
\begin{aligned}
A_{2, h}= & \int_{B_{r} \backslash B_{r / 2}}\left[F\left(\nabla u_{h}(x)\right)-F\left(\nabla u_{h}(x) \circ \nabla \Phi^{-1}(\Phi(x))\right) J \Phi(x)\right] \mathrm{d} x \\
& +\int_{B_{r} \backslash B_{r / 2}} \chi_{E_{h}}(x)\left[G\left(\nabla u_{h}(x)\right)-G\left(\nabla u_{h}(x) \circ \nabla \Phi^{-1}(\Phi(x))\right) J \Phi(x)\right] \mathrm{d} x \\
\geq & -c(p, \beta, L) \int_{B_{r} \backslash B_{r / 2}}\left(1+\chi_{E_{h}}(x)\right)\left|\nabla u_{h}(x)\right|^{p}\left(1-\left(2^{n}-1\right) \sigma\right)^{-p}\left(1+2^{n} n \sigma\right) \mathrm{d} x \\
& -c(p, \beta, L) \mu^{p}\left(1+2^{n} n \sigma\right) r^{n} \\
\geq & -C(n, p, \beta, L, \sigma) \int_{B_{r} \backslash B_{r / 2}}\left|\nabla u_{h}(x)\right|^{p} \mathrm{~d} x-c(p, \beta, L) \mu^{p}\left(1+2^{n} n \sigma\right) r^{n} \\
\geq & -C(n, p, \beta, L, \sigma, \mu)\left(\Theta+r^{n}\right) .
\end{aligned}
$$

Thus, from the above estimates we conclude that

$$
I_{1, h} \geq-C(n, p, \beta, L, \sigma, \mu)\left(\Theta+r^{n}\right) .
$$

Step 4. Estimate of $I_{2, h}$. We use the area formula for maps between rectifiable sets. To this aim, for $x \in \partial^{*} E_{h}$ denote by $T_{h, x}: \pi_{h, x} \rightarrow \mathbb{R}^{n}$ the tangential differential at $x$ of $\Phi$ along the approximate tangent space $\pi_{h, x}$ to $\partial^{*} E_{h}$, which is defined by $T_{h, x}(\tau)=\nabla \Phi(x) \circ \tau$ for all $\tau \in \pi_{h, x}$. We recall (see [5], Def. 2.68) that the $(n-1)$-dimensional jacobian of $T_{h, x}$ is given by

$$
J_{n-1} T_{h, x}=\sqrt{\operatorname{det}\left(T_{h, x}^{*} \circ T_{h, x}\right)},
$$

where $T_{h, x}^{*}$ is the adjoint of the map $T_{h, x}$. To estimate $J_{n-1} T_{h, x}$, fix $x \in \partial^{*} E_{h} \cap\left(B_{r} \backslash B_{r / 2}\right)$. Denote by $\left\{\tau_{1}, \ldots, \tau_{n-1}\right\}$ an orthonormal base for $\pi_{h, x}$, and by $L$ the $n \times(n-1)$ matrix representing $T_{h, x}$ with respect to the fixed base in $\pi_{h, x}$ and the standard base $\left\{e_{1}, \ldots, e_{n}\right\}$ in $\mathbb{R}^{n}$. From (3.6) we have

$$
L_{i j}=\nabla \Phi_{i} \cdot \tau_{j}=\frac{\varphi(|x|)}{|x|} e_{i} \cdot \tau_{j}+\left(\varphi^{\prime}(|x|)-\frac{\varphi(|x|)}{|x|}\right) \frac{x_{i}}{|x|} \frac{x \cdot \tau_{j}}{|x|}, \quad i=1, \ldots, n, j=1, \ldots, n-1 .
$$

Thus, for $j, l=1, \ldots, n-1$, we obtain

$$
\left(L^{*} \circ L\right)_{j l}=\frac{\varphi^{2}(|x|)}{|x|^{2}} \sum_{i=1}^{n}\left(e_{i} \cdot \tau_{j}\right)\left(e_{i} \cdot \tau_{l}\right)+\left(\varphi^{\prime 2}(|x|)-\frac{\varphi^{2}(|x|)}{|x|^{2}}\right) \frac{\left(x \cdot \tau_{j}\right)\left(x \cdot \tau_{l}\right)}{|x|^{2}} .
$$


Since $J_{n-1} T_{h, x}$ is invariant by rotation, in order to evaluate $\operatorname{det}\left(L^{*} \circ L\right)$ we may assume, without loss of generality, that $\tau_{j}=e_{j}$, for all $j=1, \ldots, n-1$. We deduce that

$$
L^{*} \circ L=\frac{\varphi^{2}(|x|)}{|x|^{2}} I^{(n-1)}+\left(\varphi^{\prime 2}(|x|)-\frac{\varphi^{2}(|x|)}{|x|^{2}}\right) \frac{x^{\prime} \otimes x^{\prime}}{|x|^{2}}
$$

where $I^{(n-1)}$ denotes the identity map on $\mathbb{R}^{n-1}$ and $x^{\prime}=\left(x_{1}, \ldots, x_{n-1}\right)$. With a calculation similar to the one performed to obtain (3.7), from the equality above we easily get that

$$
\operatorname{det}\left(L^{*} \circ L\right)=\left(\frac{\varphi^{2}(|x|)}{|x|^{2}}\right)^{n-1}\left[1+\frac{|x|^{2}}{\varphi^{2}(|x|)}\left(\varphi^{\prime 2}(|x|)-\frac{\varphi^{2}(|x|)}{|x|^{2}}\right) \frac{\left|x^{\prime}\right|^{2}}{|x|^{2}}\right]
$$

and so, using (3.6) we can estimate for $x \in \partial^{*} E_{h} \cap\left(B_{r} \backslash B_{r / 2}\right)$

$$
\begin{aligned}
J_{n-1} T_{h, x} & =\sqrt{\operatorname{det}\left(L^{*} \circ L\right)}=\left(\frac{\varphi(|x|)}{|x|}\right)^{n-1} \sqrt{1+\frac{|x|^{2}}{\varphi^{2}(|x|)}\left(\varphi^{\prime 2}(|x|)-\frac{\varphi^{2}(|x|)}{|x|^{2}}\right) \frac{\left|x^{\prime}\right|^{2}}{|x|^{2}}} \\
& \leq\left(\frac{\varphi(|x|)}{|x|}\right)^{n-2} \varphi^{\prime}(|x|) \leq \varphi^{\prime}(|x|) \leq 1+\sigma+2^{n}(n-1) \sigma .
\end{aligned}
$$

To estimate $I_{2, h}$, we use the area formula for maps between rectifiable sets ([5], Thm. 2.91), and we get

$$
\begin{aligned}
I_{2, h} & =P\left(E_{h}, \bar{B}_{r}\right)-P\left(\widetilde{E}_{h}, \bar{B}_{r}\right)=\int_{\partial^{*} E_{h} \cap \bar{B}_{r}} \mathrm{~d} \mathcal{H}^{n-1}-\int_{\partial^{*} E_{h} \cap \bar{B}_{r}} J_{n-1} T_{h, x} \mathrm{~d} \mathcal{H}^{n-1} \\
& =\int_{\partial^{*} E_{h} \cap \bar{B}_{r} \backslash B_{r / 2}}\left(1-J_{n-1} T_{h, x}\right) \mathrm{d} \mathcal{H}^{n-1}+\int_{\partial^{*} E_{h} \cap B_{r / 2}}\left(1-J_{n-1} T_{h, x}\right) \mathrm{d} \mathcal{H}^{n-1} .
\end{aligned}
$$

Notice that the last integral in the above formula is nonnegative since $\Phi$ is a contraction in $B_{r / 2}$, hence $J_{n-1} T_{h, x}<1$ in $B_{r / 2}$, while from (3.13) and (3.1) we have

$$
\int_{\partial^{*} E_{h} \cap \bar{B}_{r} \backslash B_{r / 2}}\left(1-J_{n-1} T_{h, x}\right) \mathrm{d} \mathcal{H}^{n-1} \geq-2^{n} n P\left(E_{h}, \bar{B}_{r}\right) \sigma \geq-2^{n} n \Theta \sigma,
$$

thus concluding that

$$
I_{2, h} \geq-c(n) \Theta \sigma
$$

Step 5. Estimate of $I_{3, h}$. We recall (3.2), (3.3), (3.7) to obtain

$$
\begin{aligned}
I_{3, h} & =\lambda_{h} \int_{E_{h} \cap B_{r} \backslash B_{r / 2}}(J \Phi(x)-1) \mathrm{d} x+\lambda_{h} \int_{E_{h} \cap B_{r / 2}}(J \Phi(x)-1) \mathrm{d} x \\
& \geq \lambda_{h} C_{1}(n)\left(\frac{\omega_{n}}{2^{n+2}}-\varepsilon\right) \sigma r^{n}-\lambda_{h}\left[1-\left(1-\left(2^{n}-1\right) \sigma\right)^{n}\right] \varepsilon r^{n} \\
& \geq \lambda_{h} \sigma r^{n}\left[C_{1}(n) \frac{\omega_{n}}{2^{n+2}}-C_{1}(n) \varepsilon-\left(2^{n}-1\right) n \varepsilon\right] .
\end{aligned}
$$

Therefore, if we choose $0<\varepsilon<\varepsilon(n)$, with $\varepsilon(n)$ depending only on the dimension, we have that

$$
I_{3, h} \geq \lambda_{h} C_{2}(n) \sigma r^{n}
$$

for some positive $C_{2}(n)$.

Step 6. Conclusion of Step 1. Estimate (3.15), together with (3.4), (3.12) and (3.14), yields

$$
\mathcal{I}_{\lambda_{h}}\left(u_{h}, E_{h}\right)-\mathcal{I}_{\lambda_{h}}\left(\tilde{u}_{h}, \widetilde{E}_{h}\right) \geq \lambda_{h} \sigma C_{3} r^{n}-C(n, p, \sigma, \mu)\left(\Theta+r^{n}\right)>0
$$


if $\lambda_{h}$ is sufficiently large. This contradicts the minimality of $\left(u_{h}, E_{h}\right)$, thus concluding the proof of the first part of Theorem 1.4.

Step 7. Conversely, if $(u, E)$ is a minimizer of $\mathcal{I}$ and $\lambda_{0}$ is as determined on Step 1, then for $\lambda>\lambda_{0}$ Steps $1-5$ ensure the existence of a minimizer $\left(u_{\lambda}, E_{\lambda}\right)$ of $\mathcal{I}_{\lambda}$ with $\left|E_{\lambda}\right|=d$. Hence, by the minimality,

$$
\mathcal{I}(u, E) \leq \mathcal{I}\left(u_{\lambda}, E_{\lambda}\right)=\mathcal{I}_{\lambda}\left(u_{\lambda}, E_{\lambda}\right) \leq \mathcal{I}_{\lambda}(u, E)=\mathcal{I}(u, E)
$$

i.e.,

$$
\mathcal{I}(u, E)=\mathcal{I}_{\lambda}\left(u_{\lambda}, E_{\lambda}\right)
$$

and so $(u, E)$ also minimizes $\mathcal{I}_{\lambda}$.

\section{Proof of Theorem 1.1}

This section is devoted to the proof of our first regularity result, stated in Theorem 1.1. The proof is obtained by establishing that the bulk energy and the perimeter of the free interface both decay on balls of radius $\rho$ as $\rho^{n-1}$, for $\rho \rightarrow 0^{+}$. We divide it in two steps: In the first we prove the decay estimate for the perimeter, and in the second we address the decay of the bulk energies.

Proof of Theorem 1.1. Let $(u, E)$ be a solution of the problem $(\mathrm{P})$.

Step 1. First decay estimate. Fix $x_{0} \in \Omega$ and let $R \leq \operatorname{dist}\left(x_{0}, \partial \Omega\right)$. Assume, without loss of generality that $0<R<1$. Here we want to prove that there exists a constant $c_{0}=c_{0}\left(n, p, \lambda_{0}, \alpha, \beta, \ell, L\right)$ such that

$$
\int_{B_{r}\left(x_{0}\right) \cap E}|\nabla u|^{p} \mathrm{~d} x+P\left(E, B_{r}\left(x_{0}\right)\right) \leq c_{0} r^{n-1},
$$

for every $0<r<R$.

First, consider $x_{0} \in \partial E \cap \Omega$ and set $\widetilde{E}:=E \backslash B_{r}\left(x_{0}\right)$ where $0<r<R$. For $\lambda_{0}$ determined in Theorem 1.4, we have

$$
\mathcal{I}_{\lambda_{0}}(u, E) \leq \mathcal{I}_{\lambda_{0}}(u, \widetilde{E})
$$

i.e.,

$$
\begin{aligned}
& \int_{\Omega}\left(F(\nabla u)+\chi_{E} G(\nabla u)\right) \mathrm{d} x+P(E, \Omega) \\
\leq & \int_{\Omega}\left(F(\nabla u)+\chi_{\widetilde{E}} G(\nabla u)\right) \mathrm{d} x+P(\widetilde{E}, \Omega)+\lambda_{0}|| \widetilde{E}|-d| .
\end{aligned}
$$

Therefore,

$$
\int_{\Omega}\left(\chi_{E}-\chi_{\widetilde{E}}\right) G(\nabla u) \mathrm{d} x+P\left(E, B_{r}\left(x_{0}\right)\right) \leq P\left(B_{r}\left(x_{0}\right)\right)+\lambda_{0}|| \widetilde{E}|-d|,
$$

and so

$$
\int_{B_{r}\left(x_{0}\right)} \chi_{E} G(\nabla u) \mathrm{d} x+P\left(E, B_{r}\left(x_{0}\right)\right) \leq c(n) r^{n-1}+c(n) \lambda_{0} r^{n} \leq c\left(n, \lambda_{0}\right) r^{n-1},
$$

since $r<1$. Lemma 2.3 implies that

$$
\alpha \frac{\ell}{2} \int_{B_{r}\left(x_{0}\right)} \chi_{E}|\nabla u|^{p} \mathrm{~d} x-c(p, \mu, \alpha, \beta, \ell, L)\left|B_{r}\left(x_{0}\right) \cap E\right|+P\left(E, B_{r}\left(x_{0}\right)\right) \leq c\left(n, \lambda_{0}\right) r^{n-1},
$$

or, equivalently,

$$
\alpha \frac{\ell}{2} \int_{B_{r}\left(x_{0}\right)} \chi_{E}|\nabla u|^{p} \mathrm{~d} x+P\left(E, B_{r}\left(x_{0}\right)\right) \leq c\left(n, \lambda_{0}\right) r^{n-1}+c(p, \mu, \alpha, \beta, \ell, L)\left|B_{r}\left(x_{0}\right) \cap E\right|
$$




$$
\leq c\left(n, p, \mu, \alpha, \beta, \ell, L, \lambda_{0}\right) r^{n-1}
$$

Therefore

$$
\min \left\{\alpha \frac{\ell}{2}, 1\right\}\left[\int_{B_{r}\left(x_{0}\right)} \chi_{E}|\nabla u|^{p} \mathrm{~d} x+P\left(E, B_{r}\left(x_{0}\right)\right)\right] \leq c\left(n, p, \mu, \alpha, \beta, \ell, L, \lambda_{0}\right) r^{n-1} .
$$

This inequality yields that

$$
\int_{B_{r}\left(x_{0}\right) \cap E}|\nabla u|^{p} \mathrm{~d} x+P\left(E, B_{r}\left(x_{0}\right)\right) \leq c_{0} r^{n-1},
$$

where we set $c_{0}:=c\left(n, p, \lambda_{0}, \alpha, \beta, \ell, L\right)$.

If $x_{0} \notin \partial E \cap \Omega$, or $B_{r}\left(x_{0}\right) \cap E$ is not empty and we argue exactly as before, or $B_{r}\left(x_{0}\right) \subset \Omega \backslash E$ and estimate (4.1) is trivially satisfied.

Step 2. Second decay estimate. Here we want to prove that there exist $\tau \in\left(0, \frac{1}{2}\right)$ and $\delta \in(0,1)$ such that for every $M>0$ there exists $h_{0} \in \mathbb{N}$ such that $\forall B\left(x_{0}, r\right) \subset \Omega$ we have

$$
\int_{B_{r}\left(x_{0}\right)}|\nabla u|^{p} \mathrm{~d} x \leq h_{0} r^{n-1} \quad \text { or } \quad \int_{B_{\tau r}\left(x_{0}\right)}|\nabla u|^{p} \mathrm{~d} x \leq M \tau^{n-\delta} \int_{B_{r}\left(x_{0}\right)}|\nabla u|^{p} \mathrm{~d} x .
$$

In order to prove (4.3), we argue by contradiction. Fix $\tau \in(0,1 / 2), \delta \in(0,1)$ and choose $M>\tau^{\delta-n}$. Suppose that for every $h \in \mathbb{N}$, there exists a ball $B_{r_{h}}\left(x_{h}\right) \subset \Omega$ such that

$$
\int_{B_{r_{h}}\left(x_{h}\right)}|\nabla u|^{p} \mathrm{~d} x>h r_{h}^{n-1}
$$

and

$$
\int_{B_{\tau r_{h}}\left(x_{h}\right)}|\nabla u|^{p} \mathrm{~d} x>M \tau^{n-\delta} \int_{B_{r_{h}}\left(x_{h}\right)}|\nabla u|^{p} \mathrm{~d} x .
$$

Note that estimates (4.2) and (4.4) yield

$$
\int_{B_{r_{h}}\left(x_{h}\right) \cap E}|\nabla u|^{p} \mathrm{~d} x+P\left(E, B_{r_{h}}\left(x_{h}\right)\right) \leq c_{0} r_{h}^{n-1}<\frac{c_{0}}{h} \int_{B_{r_{h}}\left(x_{h}\right)}|\nabla u|^{p} \mathrm{~d} x
$$

and so

$$
\int_{B_{r_{h}}\left(x_{h}\right) \cap E}|\nabla u|^{p} \mathrm{~d} x<\frac{c_{0}}{h} \int_{B_{r_{h}}\left(x_{h}\right)}|\nabla u|^{p} \mathrm{~d} x
$$

Substep 2.a. Blow-up. Set

$$
\varsigma_{h}^{p}=f_{B_{r_{h}}\left(x_{h}\right)}|\nabla u|^{p} \mathrm{~d} x
$$

and, for $y \in B_{1}(0)$, introduce the sequence of rescaled functions defined as

$$
v_{h}(y):=\frac{u\left(x_{h}+r_{h} y\right)-a_{h}}{\varsigma_{h} r_{h}}, \text { where } a_{h}:=f_{B_{r_{h}}\left(x_{h}\right)} u(x) \mathrm{d} x .
$$

We have

$$
\nabla v_{h}(y)=\frac{1}{\varsigma_{h}} \nabla u\left(x_{h}+r_{h} y\right)
$$

and a change of variable yields

$$
f_{B_{1}}\left|\nabla v_{h}(y)\right|^{p} \mathrm{~d} y=\frac{1}{\varsigma_{h}^{p}} f_{B_{r_{h}}\left(x_{h}\right)}|\nabla u(x)|^{p} \mathrm{~d} x=1 .
$$


Therefore, there exist a subsequence of $v_{h}$ (not relabeled) and $v \in W^{1, p}\left(B_{1}\right)$ such that

$$
v_{h} \rightarrow v \quad \text { weakly in } W^{1, p}\left(B_{1}\right), \text { and } v_{h} \rightarrow v \quad \text { strongly in } L^{p}\left(B_{1}\right) .
$$

Moreover, the lower semicontinuity of the norm implies

$$
f_{B_{1}}|\nabla v(y)|^{p} \mathrm{~d} y \leq \liminf _{h \rightarrow \infty} f_{B_{1}}\left|\nabla v_{h}(y)\right|^{p} \mathrm{~d} y=1 .
$$

Substep 2.b. We claim that $v_{h} \rightarrow v$ in $W_{\text {loc }}^{1, p}\left(B_{1}\right)$. Consider the sets

$$
E_{h}^{*}:=\frac{E-x_{h}}{r_{h}} \cap B_{1}
$$

Since $P\left(E_{h}^{*}, B_{1}\right)=\frac{1}{r_{h}^{n-1}} P\left(E, B_{r_{h}}\left(x_{h}\right)\right)$, by (4.6) we have that $P\left(E_{h}^{*}, B_{1}\right)$ are bounded, and so, up to the extraction of a subsequence (not relabeled), $\chi_{E_{h}^{*}} \rightarrow \chi_{E^{*}}$ in $L^{1}$ (and weakly in $B V\left(B_{1}\right)$ ) for some set of finite perimeter $E^{*} \subset B_{1}$.

Using the minimality of $(u, E)$ with respect to $(u+\varphi, E)$, for $\varphi \in W_{0}^{1, p}\left(B_{r_{h}}\left(x_{h}\right)\right)$ we obtain

$$
\int_{B_{r_{h}}\left(x_{h}\right)}\left(F(\nabla u(x))+\chi_{E} G(\nabla u(x))\right) \mathrm{d} x \leq \int_{B_{r_{h}}\left(x_{h}\right)}\left(F(\nabla u(x)+\nabla \varphi(x))+\chi_{E} G(\nabla u(x)+\nabla \varphi(x))\right) \mathrm{d} x,
$$

or, equivalently, using the change of variable $x=x_{h}+r_{h} y$, we get

$$
\begin{aligned}
& \int_{B_{1}}\left(F\left(\varsigma_{h} \nabla v_{h}(y)\right)+\chi_{E_{h}^{*}} G\left(\varsigma_{h} \nabla v_{h}(y)\right)\right) \mathrm{d} y \\
\leq & \int_{B_{1}}\left(F\left(\varsigma_{h} \nabla v_{h}(y)+\nabla \psi(y)\right)+\chi_{E_{h}^{*}} G\left(\varsigma_{h} \nabla v_{h}(y)+\nabla \psi(y)\right)\right) \mathrm{d} x
\end{aligned}
$$

for every $\psi \in W_{0}^{1, p}\left(B_{1}\right)$. Let $\eta \in C_{0}^{\infty}\left(B_{1}\right), 0 \leq \eta \leq 1$. Choosing $\psi_{h}(y)=\varsigma_{h} \eta\left(v-v_{h}\right)$ as test function in (4.10), we get

$$
\begin{aligned}
& \int_{B_{1}}\left(F\left(\varsigma_{h} \nabla v_{h}(y)\right)+\chi_{E_{h}^{*}} G\left(\varsigma_{h} \nabla v_{h}(y)\right)\right) \mathrm{d} y \\
\leq & \int_{B_{1}}\left(F\left(\varsigma_{h} \eta \nabla v(y)+\varsigma_{h}(1-\eta) \nabla v_{h}(y)+\nabla \eta \varsigma_{h}\left(v-v_{h}\right)\right)\right) \mathrm{d} y \\
& +\int_{B_{1}} \chi_{E_{h}^{*}}\left(G\left(\varsigma_{h} \eta \nabla v(y)+\varsigma_{h}(1-\eta) \nabla v_{h}(y)+\nabla \eta \varsigma_{h}\left(v-v_{h}\right)\right)\right) \mathrm{d} y \\
\leq & \int_{B_{1}}\left(F\left(\varsigma_{h} \eta \nabla v(y)+\varsigma_{h}(1-\eta) \nabla v_{h}(y)\right)\right) \mathrm{d} y+\int_{B_{1}} \chi_{E_{h}^{*}}\left(G\left(\varsigma_{h} \eta \nabla v(y)+\varsigma_{h}(1-\eta) \nabla v_{h}(y)\right)\right) \mathrm{d} y \\
& +\int_{B_{1}}\left\langle D_{\xi} F\left(\varsigma_{h} \eta \nabla v(y)+\varsigma_{h}(1-\eta) \nabla v_{h}(y)+\nabla \eta \varsigma_{h}\left(v-v_{h}\right)\right), \nabla \eta \varsigma_{h}\left(v-v_{h}\right)\right\rangle \mathrm{d} y \\
& +\int_{B_{1}} \chi_{E_{h}^{*}}\left\langle D_{\xi} G\left(\varsigma_{h} \eta \nabla v(y)+\varsigma_{h}(1-\eta) \nabla v_{h}(y)+\nabla \eta \varsigma_{h}\left(v-v_{h}\right)\right), \nabla \eta \varsigma_{h}\left(v-v_{h}\right)\right\rangle \mathrm{d} y \\
\leq & \int_{B_{1}}\left(F\left(\varsigma_{h} \eta \nabla v(y)+\varsigma_{h}(1-\eta) \nabla v_{h}(y)\right)\right) \mathrm{d} y+\int_{B_{1}} \chi_{E_{h}^{*}}\left(G\left(\varsigma_{h} \eta \nabla v(y)+\varsigma_{h}(1-\eta) \nabla v_{h}(y)\right)\right) \mathrm{d} y \\
& +c \int_{B_{1}}\left(\mu^{2}+\left|\varsigma_{h} \nabla v_{h}\right|^{2}+\left|\varsigma_{h} \nabla v\right|^{2}+\left|\varsigma_{h}\left(v-v_{h}\right)\right|^{2}\right)^{\frac{p-1}{2}}\left|\varsigma_{h}\left(v-v_{h}\right)\right| \mathrm{d} y
\end{aligned}
$$


where, in the last inequality, we used (2.2). Hence, using Hölder's inequality and the convexity of $F$ and $G$ in estimate (4.11), we obtain

$$
\begin{aligned}
& \int_{B_{1}}\left(F\left(\varsigma_{h} \nabla v_{h}(y)\right)+\chi_{E_{h}^{*}} G\left(\varsigma_{h} \nabla v_{h}(y)\right)\right) \mathrm{d} y \\
\leq & \int_{B_{1}}\left((1-\eta) F\left(\varsigma_{h} \nabla v_{h}(y)\right) \mathrm{d} y+\eta F\left(\varsigma_{h} \nabla v(y)\right)\right) \mathrm{d} y \\
& +\int_{B_{1}} \chi_{E_{h}^{*}}\left((1-\eta) G\left(\varsigma_{h} \nabla v_{h}(y)\right)+\eta G\left(\varsigma_{h} \nabla v(y)\right)\right) \mathrm{d} y \\
& +c \int_{B_{1}}\left|\varsigma_{h}\left(v-v_{h}\right)\right|^{p} \mathrm{~d} x+\varsigma_{h}^{p}\left(\int_{B_{1}} \mu^{p}+\left|\nabla v_{h}\right|^{p}+|\nabla v|^{p} \mathrm{~d} x\right)^{\frac{p-1}{p}}\left(\int_{B_{1}}\left|v-v_{h}\right|^{p} \mathrm{~d} x\right)^{\frac{1}{p}},
\end{aligned}
$$

since we may suppose that $\varsigma_{h}>1$ for $h$ large. In fact, by (4.4) and the definition of $\varsigma_{h}$, we have

$$
\varsigma_{h}^{p} \geq \frac{h}{r_{h}},
$$

and so $\varsigma_{h} \rightarrow+\infty$ as $h \rightarrow+\infty$. By virtue of (4.8), from estimate (4.12) we infer that

$$
\begin{aligned}
& \int_{B_{1}} \eta\left(F\left(\varsigma_{h} \nabla v_{h}(y)\right)+\chi_{E_{h}^{*}} G\left(\varsigma_{h} \nabla v_{h}(y)\right)\right) \mathrm{d} y \\
\leq & \int_{B_{1}}\left(\eta F\left(\varsigma_{h} \nabla v(y)\right)\right) \mathrm{d} y+\int_{B_{1}} \chi_{E_{h}^{*}}\left(\eta G\left(\varsigma_{h} \nabla v(y)\right)\right) \mathrm{d} y \\
& +c \varsigma_{h}^{p} \int_{B_{1}}\left|v-v_{h}\right|^{p}+c \varsigma_{h}^{p}\left(\int_{B_{1}}\left|v-v_{h}\right|^{p} \mathrm{~d} x\right)^{\frac{1}{p}} .
\end{aligned}
$$

Note that, by changing variable in (4.7), we have

$$
r_{h}^{n} \int_{B_{1} \cap E_{h}^{*}}\left|\nabla u\left(x_{h}+r_{h} y\right)\right|^{p} \mathrm{~d} y<\frac{c_{0}}{h} r_{h}^{n} \int_{B_{1}}\left|\nabla u\left(x_{h}+r_{h} y\right)\right|^{p} \mathrm{~d} y,
$$

and thus, by the definition of $v_{h}$,

$$
\int_{B_{1} \cap E_{h}^{*}}\left|\nabla v_{h}(y)\right|^{p} \mathrm{~d} y<\frac{c_{0}}{h} \int_{B_{1}}\left|\nabla v_{h}(y)\right|^{p} \mathrm{~d} y
$$

and, by the use of (4.8), we get

$$
\int_{B_{1} \cap E_{h}^{*}}\left|\nabla v_{h}(y)\right|^{p} \mathrm{~d} y<\frac{c_{0} \omega_{n}}{h} .
$$

Since $\chi_{E_{h}^{*}} \rightarrow \chi_{E^{*}}$ weakly in $B V\left(B_{1}\right)$, by Fatou's Lemma and (4.15) we obtain

$$
\int_{B_{1} \cap E^{*}}|\nabla v(y)|^{p} \mathrm{~d} y \leq \liminf _{h} \int_{B_{1} \cap E_{h}^{*}}\left|\nabla v_{h}(y)\right|^{p} \mathrm{~d} y=0 .
$$

Using assumption (G1) and the homogeneity of $F$ in (4.14), we get

$$
\begin{aligned}
\int_{B_{1}} \eta \varsigma_{h}^{p} F\left(\nabla v_{h}(y)\right) \mathrm{d} y \leq & \int_{B_{1}} \eta \varsigma_{h}^{p} F(\nabla v(y)) \mathrm{d} y+c \int_{B_{1}} \chi_{E_{h}^{*}}\left(\left|\varsigma_{h} \nabla v(y)\right|^{p}+\left|\varsigma_{h} \nabla v_{h}(y)\right|^{p}\right) \mathrm{d} y \\
& +c \varsigma_{h}^{p} \int_{B_{1}}\left|v-v_{h}\right|^{p} \mathrm{~d} y+c \varsigma_{h}^{p}\left(\int_{B_{1}}\left|v-v_{h}\right|^{p} \mathrm{~d} y\right)^{\frac{1}{p}}
\end{aligned}
$$


i.e.,

$$
\begin{aligned}
\int_{B_{1}} \eta F\left(\nabla v_{h}(y)\right) \mathrm{d} y \leq & \int_{B_{1}} \eta F(\nabla v(y)) \mathrm{d} y+\int_{B_{1}} \chi_{E_{h}^{*}}\left(|\nabla v(y)|^{p}+\left|\nabla v_{h}(y)\right|^{p}\right) \mathrm{d} y \\
& +c \int_{B_{1}}\left|v-v_{h}\right|^{p} \mathrm{~d} y+c\left(\int_{B_{1}}\left|v-v_{h}\right|^{p} \mathrm{~d} y\right)^{\frac{1}{p}} .
\end{aligned}
$$

Passing to the limit as $h \rightarrow+\infty$ in (4.17), by virtue of (4.16), the strong convergence of $v_{h}$ to $v$ in $L^{p}$ and the lower semicontinuity of $F$, we obtain

$$
\int_{B_{1}} \eta F(\nabla v(y)) \mathrm{d} y \leq \liminf _{h} \int_{B_{1}} \eta F\left(\nabla v_{h}(y)\right) \mathrm{d} y \leq \int_{B_{1}} \eta F(\nabla v(y)) \mathrm{d} y,
$$

that is,

$$
\lim _{h} \int_{B_{1}} \eta F\left(\nabla v_{h}(y)\right) \mathrm{d} y=\int_{B_{1}} \eta F(\nabla v(y)) \mathrm{d} y .
$$

By the strong p-convexity of $F$ and Lemma 2.1, we have

$$
\begin{gathered}
\int_{B_{1}} \eta\left|V\left(\nabla v_{h}(y)\right)-V(\nabla v(y))\right|^{2} \mathrm{~d} y \\
\leq c(p, \ell) \int_{B_{1}} \eta\left(F\left(\nabla v_{h}(y)\right)-F(\nabla v(y))\right)-\left\langle D_{\xi} F\left(\nabla v_{h}(y)\right), \eta\left(\nabla v_{h}(y)-\nabla v(y)\right)\right\rangle \mathrm{d} y .
\end{gathered}
$$

By the minimality of $(u, E)$, we get

$$
\int_{B_{r_{h}}\left(x_{h}\right)}\left\langle D_{\xi} F(\nabla u(y))+\chi_{E} D_{\xi} G(\nabla u(y)), \nabla \varphi\right\rangle \mathrm{d} x=0
$$

for every $\varphi \in W_{0}^{1, p}\left(B_{r_{h}}\left(x_{h}\right)\right)$ or, equivalently,

$$
\int_{B_{1}}\left\langle D_{\xi} F\left(\varsigma_{h} \nabla v_{h}(y)\right)+\chi_{E_{h}^{*}} D_{\xi} G\left(\varsigma_{h} \nabla v_{h}(y)\right), \nabla \psi\right\rangle \mathrm{d} x=0
$$

for every $\psi \in W_{0}^{1, p}\left(B_{1}\right)$, or still

$$
\int_{B_{1}}\left\langle D_{\xi} F\left(\varsigma_{h} \nabla v_{h}(y)\right), \nabla \psi\right\rangle \mathrm{d} y=-\int_{B_{1}}\left\langle\chi_{E_{h}^{*}} D_{\xi} G\left(\varsigma_{h} \nabla v_{h}(y)\right), \nabla \psi\right\rangle \mathrm{d} x .
$$

Then, choosing $\psi:=\eta\left(v_{h}-v\right)$ with $\eta \in C_{0}^{\infty}\left(B_{1}\right)$ as test function in (4.20), we obtain

$$
\begin{aligned}
& \int_{B_{1}}\left\langle D_{\xi} F\left(\varsigma_{h} \nabla v_{h}(y)\right), \eta\left(\nabla v_{h}-\nabla v\right)\right\rangle \mathrm{d} y \\
= & -\int_{B_{1}}\left\langle D_{\xi} F\left(\varsigma_{h} \nabla v_{h}(y)\right), \nabla \eta\left(v_{h}-v\right)\right\rangle \mathrm{d} y \\
& -\int_{B_{1}} \chi_{E_{h}^{*}}\left\langle D_{\xi} G\left(\varsigma_{h} \nabla v_{h}(y)\right), \eta\left(\nabla v_{h}-\nabla v\right)\right\rangle \mathrm{d} y \\
& -\int_{B_{1}} \chi_{E_{h}^{*}}\left\langle D_{\xi} G\left(\varsigma_{h} \nabla v_{h}(y)\right), \nabla \eta\left(v_{h}-v\right)\right\rangle \mathrm{d} y .
\end{aligned}
$$

Using estimates (2.2) for $D_{\xi} F$ and $D_{\xi} G,(4.21)$ yields

$$
\left|\int_{B_{1}}\left\langle D_{\xi} F\left(\varsigma_{h} \nabla v_{h}(y)\right), \eta\left(\nabla v_{h}-\nabla v\right)\right\rangle \mathrm{d} y\right|
$$




$$
\begin{aligned}
\leq & c(p, \beta, L) \int_{B_{1}}\left|\varsigma_{h} \nabla v_{h}(y)\right|^{p-1}|\nabla \eta|\left|v_{h}-v\right| \mathrm{d} y \\
& +c(p, \beta, L) \int_{B_{1} \cap E_{h}^{*}}\left|\varsigma_{h} \nabla v_{h}(y)\right|^{p-1}|\eta|\left|\nabla v_{h}-\nabla v\right| \mathrm{d} y .
\end{aligned}
$$

By the homogeneity of $F$, Hölder's inequality, (4.8) and (4.15), (4.22) implies that

$$
\begin{aligned}
& \left|\int_{B_{1}}\left\langle D_{\xi} F\left(\nabla v_{h}(y)\right), \eta\left(\nabla v_{h}-\nabla v\right)\right\rangle \mathrm{d} y\right| \\
\leq & c\left(p, \beta, L,\|\nabla \eta\|_{\infty}\right)\left(\int_{B_{1}}\left|\nabla v_{h}(y)\right|^{p} \mathrm{~d} y\right)^{\frac{p-1}{p}}\left(\int_{B_{1}}\left|v_{h}-v\right|^{p} \mathrm{~d} y\right)^{\frac{1}{p}} \\
& +c\left(p, \beta, L,\|\eta\|_{\infty}\right)\left(\int_{B_{1} \cap E_{h}^{*}}\left|\nabla v_{h}(y)\right|^{p} \mathrm{~d} y\right)^{\frac{p-1}{p}}\left(\int_{B_{1}}\left|\nabla v_{h}\right|^{p}+|\nabla v|^{p} \mathrm{~d} y\right)^{\frac{1}{p}} \\
\leq & c\left(n, p, \beta, L,\|\nabla \eta\|_{\infty}\right)\left(\int_{B_{1}}\left|v_{h}-v\right|^{p} \mathrm{~d} y\right)^{\frac{1}{p}}+c\left(p, \beta, L,\|\eta\|_{\infty}\right)\left(\frac{c_{0}}{h}\right)^{\frac{p-1}{p}} .
\end{aligned}
$$

Since $v_{h}$ converge strongly to $v$ in $L^{p}\left(B_{1}\right)$, passing to the limit as $h \rightarrow \infty$ in (4.23), we get

$$
\lim _{h \rightarrow+\infty}\left|\int_{B_{1}}\left\langle D_{\xi} F\left(\nabla v_{h}(y)\right), \eta\left(\nabla v_{h}-\nabla v\right)\right\rangle \mathrm{d} y\right|=0 .
$$

Passing to the limit in (4.19) and using (4.18) and (4.24), we obtain

$$
\lim _{h \rightarrow+\infty} \int_{B_{1}} \eta\left|V\left(\nabla v_{h}(y)\right)-V(\nabla v(y))\right|^{2} \mathrm{~d} y=0,
$$

which, by Lemma 2.1, implies that

$$
\lim _{h \rightarrow+\infty} \int_{B_{1}} \eta\left(\mu^{2}+\left|\nabla v_{h}(y)\right|^{2}+|\nabla v(y)|^{2}\right)^{\frac{p-2}{2}}\left|\nabla v_{h}(y)-\nabla v(y)\right|^{2} \mathrm{~d} y=0 .
$$

In the case $p \geq 2$, one can easily check that (4.25) implies

$$
v_{h} \rightarrow v \quad \text { strongly in } W_{\mathrm{loc}}^{1, p}\left(B_{1}\right) .
$$

In the case $1<p<2$, it suffices to observe that Hölder's inequality with exponents $\frac{2}{p}$ and $\frac{2}{2-p}$ yields

$$
\begin{aligned}
& \int_{B_{1}} \eta\left|\nabla v_{h}-\nabla v\right|^{p} \mathrm{~d} x \\
\leq & \left(\int_{B_{1}} \eta\left(\mu^{2}+\left|\nabla v_{h}\right|^{2}+|\nabla v|^{2}\right)^{\frac{p-2}{2}}\left|\nabla v_{h}-\nabla v\right|^{2} \mathrm{~d} x\right)^{\frac{p}{2}}\left(\int_{B_{1}} \eta\left(\mu^{2}+\left|\nabla v_{h}\right|^{2}+|\nabla v|^{2}\right)^{\frac{p}{2}} \mathrm{~d} x\right)^{\frac{2-p}{2}} \\
\leq & c\left(\int_{B_{1}} \eta\left(\mu^{2}+\left|\nabla v_{h}\right|^{2}+|\nabla v|^{2}\right)^{\frac{p-2}{2}}\left|\nabla v_{h}-\nabla v\right|^{2} \mathrm{~d} x\right)^{\frac{p}{2}},
\end{aligned}
$$

where we used (4.8). Hence, also in this case, by (4.25) we conclude that

$$
v_{h} \rightarrow v \quad \text { strongly in } W_{\text {loc }}^{1, p}\left(B_{1}\right),
$$

and this asserts the claim. 
Substep 2.c. Reaching a contradiction. Notice that (4.5) can be written as

$$
f_{B_{\tau r_{h}}\left(x_{h}\right)}|\nabla u|^{p} \mathrm{~d} x>M \tau^{-\delta} f_{B_{r_{h}}\left(x_{h}\right)}|\nabla u|^{p} \mathrm{~d} x
$$

or, equivalently,

$$
\frac{1}{\varsigma_{h}^{p}} f_{B_{\tau r_{h}}\left(x_{h}\right)}|\nabla u|^{p} \mathrm{~d} x>M \tau^{-\delta},
$$

by the definition of $\varsigma_{h}$. By the change of variable $x=x_{h}+r_{h} y$ and the definition of $v_{h}$, from (4.27) we infer that

$$
\frac{1}{\varsigma_{h}^{p}} f_{B_{\tau}}\left|\varsigma_{h} \nabla v_{h}\right|^{p} \mathrm{~d} x>M \tau^{-\delta},
$$

i.e.,

$$
f_{B_{\tau}}\left|\nabla v_{h}\right|^{p} \mathrm{~d} x>M \tau^{-\delta}
$$

By virtue of the strong convergence of $v_{h}$ to $v$ in $W_{\text {loc }}^{1, p}\left(B_{1}\right)$ and $(4.9)$, we have that

$$
\lim _{h} f_{B_{\tau}}\left|\nabla v_{h}\right|^{p}=f_{B_{\tau}}|\nabla v|^{p} \leq \frac{1}{\tau^{n}}
$$

Clearly, (4.29) contradicts (4.28) because $M>\tau^{\delta-n}$.

Step 3. Conclusion. We conclude that if $(u, E)$ is a solution of $(\mathrm{P})$, then there exist $\tau \in\left(0, \frac{1}{2}\right)$ and $\delta \in(0,1)$ such that, setting $M=1$, there exists $h_{0} \in \mathbb{N}$ with the property that whenever $B_{r}(x) \subset \Omega$, then

$$
\int_{B_{r}\left(x_{0}\right)}|\nabla u|^{p} \mathrm{~d} x \leq h_{0} r^{n-1} \quad \text { or } \quad \int_{B_{\tau_{r}}\left(x_{0}\right)}|\nabla u|^{p} \mathrm{~d} x \leq \tau^{n-\delta} \int_{B_{r}\left(x_{0}\right)}|\nabla u|^{p} \mathrm{~d} x .
$$

Hence,

$$
\int_{B_{\tau r}\left(x_{0}\right)}|\nabla u|^{p} \mathrm{~d} x \leq \tau^{n-\delta} \int_{B_{r}\left(x_{0}\right)}|\nabla u|^{p} \mathrm{~d} x+h_{0} r^{n-1},
$$

and using Lemma 2.4 with $\varphi(\rho):=\int_{B_{\rho}\left(x_{0}\right)}|\nabla u|^{p} \mathrm{~d} x, \gamma=n-\delta$ and $\beta=n-1$, we obtain that

$$
\int_{B_{\rho}\left(x_{0}\right)}|\nabla u|^{p} \mathrm{~d} x \leq c\left\{\left(\frac{\rho}{r}\right)^{n-1} \int_{B_{r}\left(x_{0}\right)}|\nabla u|^{p} \mathrm{~d} x+h_{0} \rho^{n-1}\right\},
$$

for every $0<\rho<r \leq R$, and so

$$
\int_{B_{\rho}\left(x_{0}\right)}|\nabla u|^{p} \mathrm{~d} x \leq C \rho^{n-1}
$$

By Hölder's inequality

$$
\int_{B_{\rho}\left(x_{0}\right)}|\nabla u| \mathrm{d} x \leq c\left(\int_{B_{\rho}\left(x_{0}\right)}|\nabla u|^{p} \mathrm{~d} x\right)^{\frac{1}{p}} \rho^{\frac{n}{p^{\prime}}} \leq C \rho^{\frac{n-1}{p}+\frac{n}{p^{\prime}}}=C \rho^{n-\frac{1}{p}} .
$$

Theorem 2.2 yields that, at least, $u$ is locally Hölder continuous with exponent $\frac{1}{p^{\prime}}$. At this point, the equivalence between reduced boundary and topological boundary can be obtained arguing as in ([4], Thm. 2.2), with the obvious modifications. 


\section{Proof of Theorem 1.2 - Full Regularity}

This section is devoted to the proof of the full regularity result stated in Theorem 1.2. The key point is to prove that if the ratio $\frac{\beta}{\alpha+1}$, where $\alpha$ and $\beta$ are the parameters appearing in hypotheses (G1) and (G2), is sufficiently small then $\int_{B_{\rho}}|\nabla u|^{p}$ decays as $\rho^{n-1+\delta}$.

Proof of Theorem 1.2.

Step 1. Let $(u, E)$ be a minimal configuration of problem $(\mathrm{P})$. We first show that $u \in C_{\mathrm{loc}}^{0,1 / p^{\prime}+\delta}(\Omega)$ for some positive $\delta$, with $p^{\prime}=\frac{p}{p-1}$. Fix $x \in \Omega$ and a ball $B_{r}(x) \subset \subset \Omega$. Assume, without loss of generality, that $x=0$ and $r<1$. In what follows, we will omit the dependence on the center simply denoting by $B_{r}$ the ball $B_{r}(0)$. By Theorem 1.4, we have that $(u, E)$ is a minimizer of problem (1.5) for $\lambda$ sufficiently large. Let $v$ be the minimizer of

$$
w \in W^{1, p}\left(B_{r}\right) \mapsto \int_{B_{r}}(F+G)(\nabla w) \mathrm{d} x,
$$

satisfying the boundary condition $v=u$ on $\partial B_{r}$. Then

$$
\int_{B_{r}}\left(D_{\xi} F(\nabla u)+\chi_{E} D_{\xi} G(\nabla u)\right) \cdot \nabla \varphi \mathrm{d} x=0
$$

and

$$
\int_{B_{r}} D_{\xi}(F+G)(\nabla v) \cdot \nabla \varphi \mathrm{d} x=0
$$

for all $\varphi \in W_{0}^{1, p}\left(B_{r}\right)$. Note that assumptions (F1)-(F2) and (G1)-(G2) imply that the integrand $F+G$ satisfies

$$
\begin{aligned}
0 & \leq(F+G)(\xi) \leq \widetilde{L}\left(\mu^{2}+|\xi|^{2}\right)^{\frac{p}{2}} \\
\int_{\Omega}(F+G)(\xi+\nabla \varphi) \mathrm{d} x & \geq \int_{\Omega}\left((F+G)(\xi)+\widetilde{\ell}\left(\mu^{2}+|\xi|^{2}+|\nabla \varphi|^{2}\right)^{\frac{p-2}{2}}|\nabla \varphi|^{2}\right) \mathrm{d} x,
\end{aligned}
$$

and (see (2.3))

$$
\left\langle D_{\xi}(F+G)(\xi)-D_{\xi}(F+G)(\eta), \xi-\eta\right\rangle \geq c(p) \widetilde{\ell}|V(\xi)-V(\eta)|^{2},
$$

with growth and coercivity constants $\widetilde{L}, \tilde{\ell}$ such that

$$
\tilde{L} \leq(\beta+1) L \quad \text { and } \quad \tilde{\ell} \geq(\alpha+1) \ell .
$$

By virtue of (H1) and (H2), we can apply Theorem 2.1 and (2.10) to $H=F+G$, to obtain that for all $0<\varrho<\frac{r}{2}$

$$
\begin{aligned}
\int_{B_{\varrho}}\left(\mu^{2}+|\nabla v|^{2}\right)^{\frac{p}{2}} \mathrm{~d} x & \leq\left|B_{\varrho}\right| \sup _{B_{\varrho}}\left(\mu^{2}+|\nabla v|^{2}\right)^{\frac{p}{2}} \leq c_{n} \varrho^{n} \sup _{B_{\frac{r}{2}}}\left(\mu^{2}+|\nabla v|^{2}\right)^{\frac{p}{2}} \\
& \leq c\left(\frac{\widetilde{L}}{\widetilde{\ell}}\right)^{\sigma}\left(\frac{\varrho}{r}\right)^{n} \int_{B_{r}}\left(\mu^{2}+|\nabla v|^{2}\right)^{\frac{p}{2}} \mathrm{~d} x
\end{aligned}
$$

for some constants $c=c(n, p) \geq 1$ and $\sigma=\frac{2 n}{p}$. On the other hand if $\frac{r}{2} \leq \varrho<r$, one easily gets that

$$
\int_{B_{\varrho}}\left(\mu^{2}+|\nabla v|^{2}\right)^{\frac{p}{2}} \mathrm{~d} x \leq 2^{n} \frac{\varrho^{n}}{r^{n}} \int_{B_{r}}\left(\mu^{2}+|\nabla v|^{2}\right)^{\frac{p}{2}} \mathrm{~d} x .
$$


Therefore estimate (5.3) holds for every $0<\varrho<r$. Subtracting (5.2) from (5.1), we obtain

$$
\int_{B_{r}}\left(D_{\xi}(F+G)(\nabla u)-D_{\xi}(F+G)(\nabla v)\right) \cdot \nabla \varphi \mathrm{d} x-\int_{B_{r} \backslash E} D_{\xi} G(\nabla u) \cdot \nabla \varphi \mathrm{d} x=0,
$$

or, equivalently,

$$
\int_{B_{r}}\left(D_{\xi}(F+G)(\nabla u)-D_{\xi}(F+G)(\nabla v)\right) \cdot \nabla \varphi \mathrm{d} x=\int_{B_{r} \backslash E} D_{\xi} G(\nabla u) \cdot \nabla \varphi \mathrm{d} x .
$$

Next, we treat separately the cases $p \geq 2$ and $1<p<2$.

Case $p \geq 2$. Set $\varphi:=u-v$ in (5.4). In (5.4) we use (H3) and Lemma 2.1 in the left hand side, the second condition in (2.2) and Hölder's inequality in the right hand side, thus obtaining

$$
\begin{aligned}
& c_{p, n} \tilde{\ell} \int_{B_{r}}\left(\mu^{2}+|\nabla u|^{2}+|\nabla v|^{2}\right)^{\frac{p-2}{2}}|\nabla u-\nabla v|^{2} \mathrm{~d} x \leq c_{p} \beta L \int_{B_{r}}\left(\mu^{2}+|\nabla u|^{2}\right)^{\frac{p-1}{2}}|\nabla u-\nabla v| \mathrm{d} x \\
\leq & c_{p} \beta L\left(\int_{B_{r}}\left(\mu^{2}+|\nabla u|^{2}\right)^{\frac{p}{2}} \mathrm{~d} x\right)^{\frac{1}{2}}\left(\int_{B_{r}}\left(\mu^{2}+|\nabla u|^{2}\right)^{\frac{p-2}{2}}|\nabla u-\nabla v|^{2} \mathrm{~d} x\right)^{\frac{1}{2}} \\
\leq & c_{p} \beta L\left(\int_{B_{r}}\left(\mu^{2}+|\nabla u|^{2}\right)^{\frac{p}{2}} \mathrm{~d} x\right)^{\frac{1}{2}}\left(\int_{B_{r}}\left(\mu^{2}+|\nabla u|^{2}+|\nabla v|^{2}\right)^{\frac{p-2}{2}}|\nabla u-\nabla v|^{2} \mathrm{~d} x\right)^{\frac{1}{2}}
\end{aligned}
$$

where, in the last inequality, we used that $p \geq 2$. Hence

$$
\int_{B_{r}}\left(\mu^{2}+|\nabla u|^{2}+|\nabla v|^{2}\right)^{\frac{p-2}{2}}|\nabla u-\nabla v|^{2} \mathrm{~d} x \leq c_{p, n}\left(\frac{\beta L}{\widetilde{\ell}}\right)^{2} \int_{B_{r}}\left(\mu^{2}+|\nabla u|^{2}\right)^{\frac{p}{2}} \mathrm{~d} x .
$$

By virtue of (5.5), one has that for $0<\rho<r$

$$
\begin{aligned}
\int_{B_{\varrho}}|\nabla u-\nabla v|^{p} \mathrm{~d} x & \leq c_{p} \int_{B_{\varrho}}\left(\mu^{2}+|\nabla u|^{2}+|\nabla v|^{2}\right)^{\frac{p-2}{2}}|\nabla u-\nabla v|^{2} \mathrm{~d} x \\
& \leq c_{p, n}\left(\frac{\beta L}{\widetilde{\ell}}\right)^{2} \int_{B_{r}}\left(\mu^{2}+|\nabla u|^{2}\right)^{\frac{p}{2}} \mathrm{~d} x,
\end{aligned}
$$

therefore, from (5.3) and (5.6), we get

$$
\begin{aligned}
\left(\int_{B_{\varrho}}|\nabla u|^{p} \mathrm{~d} x\right)^{1 / p} \leq & \left(\int_{B_{\varrho}}|\nabla u-\nabla v|^{p} \mathrm{~d} x\right)^{1 / p}+\left(\int_{B_{\varrho}}|\nabla v|^{p} \mathrm{~d} x\right)^{1 / p} \\
\leq & c_{n, p}\left(\frac{\beta L}{\widetilde{\ell}}\right)^{\frac{2}{p}}\left(\int_{B_{r}}\left(\mu^{2}+|\nabla u|^{2}\right)^{\frac{p}{2}} \mathrm{~d} x\right)^{1 / p} \\
& +c_{n, p}\left(\frac{\widetilde{L}}{\widetilde{\ell}}\right)^{\frac{\sigma}{p}}\left(\frac{\varrho}{r}\right)^{n / p}\left(\int_{B_{r}}\left(\mu^{2}+|\nabla v|^{2}\right)^{\frac{p}{2}} \mathrm{~d} x\right)^{1 / p} .
\end{aligned}
$$

By Lemma 2.3 applied for $H=F+G$ and by the minimality of $v$, we have

$$
\begin{aligned}
\int_{B_{r}}\left(\mu^{2}+|\nabla v|^{2}\right)^{\frac{p}{2}} \mathrm{~d} x & \leq \frac{2}{\widetilde{\ell}} \int_{B_{r}}(F+G)(\nabla v) \mathrm{d} x+c(n, p, \widetilde{L}, \widetilde{\ell}, \mu) r^{n} \\
& \leq \frac{2}{\widetilde{\ell}} \int_{B_{r}}(F+G)(\nabla u) \mathrm{d} x+c(n, p, \widetilde{L}, \widetilde{\ell}, \mu) r^{n}
\end{aligned}
$$




$$
\leq \frac{2 \widetilde{L}}{\widetilde{\ell}} \int_{B_{r}}\left(\mu^{2}+|\nabla u|^{2}\right)^{\frac{p}{2}} \mathrm{~d} x+c(n, p, \widetilde{L}, \widetilde{\ell}, \mu) r^{n},
$$

where in last line we used the growth assumption (H1). Combining (5.7) and (5.8), we obtain, for all $0<\varrho<r$, that

$$
\begin{aligned}
\left(\int_{B_{\varrho}}|\nabla u|^{p} \mathrm{~d} x\right)^{\frac{1}{p}} \leq & c_{n, p}\left(\frac{\beta L}{\widetilde{\ell}}\right)^{\frac{2}{p}}\left(\int_{B_{r}}\left(\mu^{2}+|\nabla u|^{2}\right)^{\frac{p}{2}} \mathrm{~d} x\right)^{\frac{1}{p}} \\
& +c_{n, p}\left(\frac{\widetilde{L}}{\widetilde{\ell}}\right)^{\frac{\sigma+1}{p}}\left(\frac{\varrho}{r}\right)^{\frac{n}{p}}\left(\int_{B_{r}}\left(\mu^{2}+|\nabla u|^{2}\right)^{\frac{p}{2}} \mathrm{~d} x\right)^{\frac{1}{p}}+c(n, p, \widetilde{L}, \widetilde{\ell}, \mu) \varrho^{\frac{n}{p}} \\
= & c_{n, p}\left[\left(\frac{\beta L}{\widetilde{\ell}}\right)^{\frac{2}{p}}+\left(\frac{\widetilde{L}}{\widetilde{\ell}}\right)^{\frac{\sigma+1}{p}}\left(\frac{\varrho}{r}\right)^{\frac{n}{p}}\right]\left(\int_{B_{r}}\left(\mu^{2}+|\nabla u|^{2}\right)^{\frac{p}{2}} \mathrm{~d} x\right)^{\frac{1}{p}} \\
& +c(n, p, \widetilde{L}, \widetilde{\ell}, \mu) \varrho^{\frac{n}{p}},
\end{aligned}
$$

and therefore the following estimate holds

$$
\begin{aligned}
\int_{B_{\varrho}}|\nabla u|^{p} \mathrm{~d} x \leq & c_{n, p}\left[\left(\frac{\beta L}{\widetilde{\ell}}\right)^{\frac{2}{p}}+\left(\frac{\widetilde{L}}{\widetilde{\ell}}\right)^{\frac{\sigma+1}{p}}\left(\frac{\varrho}{r}\right)^{\frac{n}{p}}\right]^{p} \int_{B_{r}}\left(\mu^{2}+|\nabla u|^{2}\right)^{\frac{p}{2}} \mathrm{~d} x \\
& +c(n, p, \widetilde{L}, \widetilde{\ell}, \mu) \varrho^{n} .
\end{aligned}
$$

for every $0<\rho<r$.

Case $1<p<2$. As before, we choose $\varphi=u-v$ in (5.4). In (5.4) we use (H3) and Lemma 2.1 in the left hand side, the second condition in (2.2) and Hölder's inequality in the right hand side to obtain

$$
\begin{aligned}
& c_{n, p} \tilde{\ell} \int_{B_{r}}\left(\mu^{2}+|\nabla u|^{2}+|\nabla v|^{2}\right)^{\frac{p-2}{2}}|\nabla u-\nabla v|^{2} \mathrm{~d} x \leq c_{p} \beta L \int_{B_{r}}\left(\mu^{2}+|\nabla u|^{2}\right)^{\frac{p-1}{2}}|\nabla u-\nabla v| \mathrm{d} x \\
& \leq c_{p} \beta L \int_{B_{r}}\left(\mu^{2}+|\nabla u|^{2}+|\nabla v|^{2}\right)^{\frac{p-1}{2}}|\nabla u-\nabla v| \mathrm{d} x \\
& \leq c_{p} \beta L\left(\int_{B_{r}}\left(\mu^{2}+|\nabla u|^{2}+|\nabla v|^{2}\right)^{\frac{p}{2}} \mathrm{~d} x\right)^{\frac{1}{2}}\left(\int_{B_{r}}\left(\mu^{2}+|\nabla u|^{2}+|\nabla v|^{2}\right)^{\frac{p-2}{2}}|\nabla u-\nabla v|^{2} \mathrm{~d} x\right)^{\frac{1}{2}}
\end{aligned}
$$

and so

$$
\int_{B_{r}}\left(\mu^{2}+|\nabla u|^{2}+|\nabla v|^{2}\right)^{\frac{p-2}{2}}|\nabla u-\nabla v|^{2} \mathrm{~d} x \leq c_{n, p}\left(\frac{\beta L}{\widetilde{\ell}}\right)^{2} \int_{B_{r}}\left(\mu^{2}+|\nabla u|^{2}+|\nabla v|^{2}\right)^{\frac{p}{2}} \mathrm{~d} x
$$

On the other hand, for $1<p<2$, Hölder's inequality with exponents $\frac{2}{p}$ and $\frac{2}{2-p}$ yields

$$
\begin{aligned}
& \int_{B_{\varrho}}|\nabla u-\nabla v|^{p} \mathrm{~d} x \\
\leq & \left(\int_{B_{\varrho}}\left(\mu^{2}+|\nabla u|^{2}+|\nabla v|^{2}\right)^{\frac{p-2}{2}}|\nabla u-\nabla v|^{2} \mathrm{~d} x\right)^{\frac{p}{2}}\left(\int_{B_{\varrho}}\left(\mu^{2}+|\nabla u|^{2}+|\nabla v|^{2}\right)^{\frac{p}{2}} \mathrm{~d} x\right)^{\frac{2-p}{2}} \\
\leq & c_{n, p}\left(\frac{\beta L}{\widetilde{\ell}}\right)^{p}\left(\int_{B_{r}}\left(\mu^{2}+|\nabla u|^{2}+|\nabla v|^{2}\right)^{\frac{p}{2}} \mathrm{~d} x\right)^{\frac{p}{2}}\left(\int_{B_{\varrho}}\left(\mu^{2}+|\nabla u|^{2}+|\nabla v|^{2}\right)^{\frac{p}{2}} \mathrm{~d} x\right)^{\frac{2-p}{2}}
\end{aligned}
$$




$$
=c_{n, p}\left(\frac{\beta L}{\widetilde{\ell}}\right)^{p} \int_{B_{r}}\left(\mu^{2}+|\nabla u|^{2}+|\nabla v|^{2}\right)^{\frac{p}{2}} \mathrm{~d} x,
$$

where we used (5.10). From (5.3) and (5.11), we get

$$
\begin{aligned}
\left(\int_{B_{\varrho}}|\nabla u|^{p} \mathrm{~d} x\right)^{1 / p} \leq & \left(\int_{B_{\varrho}}|\nabla u-\nabla v|^{p} \mathrm{~d} x\right)^{1 / p}+\left(\int_{B_{\varrho}}|\nabla v|^{p} \mathrm{~d} x\right)^{1 / p} \\
\leq & c_{n, p}\left(\frac{\beta L}{\widetilde{\ell}}\right)\left(\int_{B_{r}}\left(\mu^{2}+|\nabla u|^{2}+|\nabla v|^{2}\right)^{\frac{p}{2}} \mathrm{~d} x\right)^{1 / p} \\
& +c_{n, p}\left(\frac{\widetilde{L}}{\widetilde{\ell}}\right)^{\frac{\sigma}{p}}\left(\frac{\varrho}{r}\right)^{n / p}\left(\int_{B_{r}}\left(\mu^{2}+|\nabla v|^{2}\right)^{\frac{p}{2}} \mathrm{~d} x\right)^{1 / p} \\
\leq & c_{n, p}\left(\frac{\beta L}{\widetilde{\ell}}\right)\left(\int_{B_{r}}\left(\mu^{2}+|\nabla u|^{2}\right)^{\frac{p}{2}} \mathrm{~d} x\right)^{1 / p} \\
& +c_{n, p}\left[\left(\frac{\beta L}{\widetilde{\ell}}\right)+\left(\frac{\widetilde{L}}{\widetilde{\ell}}\right)^{\frac{\sigma}{p}}\left(\frac{\varrho}{r}\right)^{n / p}\right]\left(\int_{B_{r}}\left(\mu^{2}+|\nabla v|^{2}\right)^{\frac{p}{2}} \mathrm{~d} x\right)^{1 / p} .
\end{aligned}
$$

By virtue of (5.8), that holds for all $p>1$, from estimate (5.12) we obtain

$$
\begin{aligned}
\left(\int_{B_{\varrho}}|\nabla u|^{p} \mathrm{~d} x\right)^{1 / p} \leq & c_{n, p}\left(\frac{\beta L}{\widetilde{\ell}}\right)\left(\int_{B_{r}}\left(\mu^{2}+|\nabla u|^{2}\right)^{\frac{p}{2}} \mathrm{~d} x\right)^{1 / p} \\
& +c_{n, p}\left[\left(\frac{\beta L}{\widetilde{\ell}}\right)+\left(\frac{\widetilde{L}}{\widetilde{\ell}}\right)^{\frac{\sigma}{p}}\left(\frac{\varrho}{r}\right)^{n / p}\right]\left(\frac{\widetilde{L}}{\widetilde{\ell}}\right)^{\frac{1}{p}}\left(\int_{B_{r}}\left(\mu^{2}+|\nabla u|^{2}\right)^{\frac{p}{2}} \mathrm{~d} x\right)^{1 / p} \\
& +c(n, p, \widetilde{L}, \widetilde{\ell}, \mu) r^{\frac{n}{p}} \\
\leq & c_{n, p}\left[\left(\frac{\beta L}{\widetilde{\ell}}\right)\left(\frac{\widetilde{L}}{\widetilde{\ell}}\right)^{\frac{1}{p}}+\left(\frac{\widetilde{L}}{\widetilde{\ell}}\right)^{\frac{\sigma+1}{p}}\left(\frac{\varrho}{r}\right)^{n / p}\right]\left(\int_{B_{r}}\left(\mu^{2}+|\nabla u|^{2}\right)^{\frac{p}{2}} \mathrm{~d} x\right)^{1 / p} \\
& +c(n, p, \widetilde{L}, \widetilde{\ell}, \mu) r^{\frac{n}{p}} .
\end{aligned}
$$

Therefore

$$
\begin{aligned}
\int_{B_{\varrho}}|\nabla u|^{p} \mathrm{~d} x \leq & c_{n, p}\left[\left(\frac{\beta L}{\widetilde{\ell}}\right)\left(\frac{\widetilde{L}}{\widetilde{\ell}}\right)^{\frac{1}{p}}+\left(\frac{\widetilde{L}}{\widetilde{\ell}}\right)^{\frac{\sigma+1}{p}}\left(\frac{\varrho}{r}\right)^{n / p}\right]^{p} \int_{B_{r}}\left(\mu^{2}+|\nabla u|^{2}\right)^{\frac{p}{2}} \mathrm{~d} x \\
& +c(n, p, \widetilde{L}, \widetilde{\ell}, \mu) r^{n} .
\end{aligned}
$$

Hence, both estimates (5.9) and (5.14) can be written as

$$
\int_{B_{\varrho}}|\nabla u|^{p} \mathrm{~d} x \leq c_{n, p}\left[\zeta+\left(\frac{\widetilde{L}}{\widetilde{\ell}}\right)^{\frac{\sigma+1}{p}}\left(\frac{\rho}{r}\right)^{n / p}\right]^{p} \int_{B_{r}}|\nabla u|^{p} \mathrm{~d} x+c(n, p, \widetilde{L}, \widetilde{\ell}, \mu) r^{n},
$$

where

$$
\zeta:= \begin{cases}\left(\frac{\beta L}{\tilde{\ell}}\right)^{\frac{2}{p}} & \text { if } p \geq 2, \\ \left(\frac{\beta L}{\tilde{\ell}}\right)\left(\frac{\widetilde{L}}{\tilde{\ell}}\right)^{\frac{1}{p}} & \text { if } 1<p<2 .\end{cases}
$$


We find the largest $\zeta<1$ for which there exists $\vartheta<1$ such that

$$
c_{n, p}\left(\zeta+\left(\frac{\widetilde{L}}{\widetilde{\ell}}\right)^{\frac{\sigma+1}{p}} \vartheta^{n / p}\right)^{p}=\vartheta^{n-1} .
$$

This equality is equivalent to

$$
\zeta=\frac{\vartheta^{(n-1) / p}}{c^{\frac{1}{p}}}-\left(\frac{\widetilde{L}}{\widetilde{\ell}}\right)^{\frac{\sigma+1}{p}} \vartheta^{n / p}=: f(\vartheta),
$$

where, for simplicity, we set $c=c_{n, p}, c>1$. Note that such $\vartheta, \zeta \in[0,1)$ exist. Indeed

$$
\frac{d f}{d \vartheta}(\vartheta)=\frac{1}{p} \vartheta^{\frac{n}{p}-1}\left(\frac{n-1}{c^{\frac{1}{p}}} \vartheta^{-\frac{1}{p}}-n\left(\frac{\widetilde{L}}{\widetilde{\ell}}\right)^{\frac{\sigma+1}{p}}\right),
$$

and so

$$
\frac{d f}{d \vartheta}(\vartheta)=0 \quad \Leftrightarrow \quad \vartheta=\frac{1}{c}\left(\frac{n-1}{n}\right)^{p}\left(\frac{\widetilde{L}}{\widetilde{\ell}}\right)^{-(\sigma+1)} .
$$

Set

$$
\vartheta_{0}:=\frac{1}{c}\left(\frac{n-1}{n}\right)^{p}\left(\frac{\widetilde{L}}{\widetilde{\ell}}\right)^{-(\sigma+1)} \quad \text { and } \quad \zeta_{0}:=f\left(\vartheta_{0}\right) .
$$

Since $\tilde{\ell} \leq \widetilde{L}$ and $c \geq 1$ it follows that $\vartheta_{0} \in(0,1)$ and

$$
f\left(\vartheta_{0}\right)=\max _{\vartheta \in[0,1]} f(\vartheta) .
$$

Moreover,

$$
\begin{aligned}
\zeta_{0} & =\frac{\vartheta_{0}^{(n-1) p}}{c^{\frac{1}{p}}}-\left(\frac{\widetilde{L}}{\widetilde{\ell}}\right)^{\frac{\sigma+1}{p}} \vartheta_{0}^{\frac{n}{p}}=\vartheta_{0}^{\frac{n}{p}}\left(\frac{\vartheta_{0}^{-\frac{1}{p}}}{c^{\frac{1}{p}}}-\left(\frac{\widetilde{L}}{\widetilde{\ell}}\right)^{\frac{\sigma+1}{p}}\right)^{\frac{n}{p}}\left(\frac{n}{n-1}-1\right)\left(\frac{\widetilde{L}}{\widetilde{\ell}}\right)^{\frac{\sigma+1}{p}}=\vartheta_{0}^{\frac{n}{p}}\left(\frac{1}{n-1}\right)\left(\frac{\widetilde{L}}{\widetilde{\ell}}\right)^{\frac{\sigma+1}{p}}
\end{aligned}
$$

We write

$$
\zeta_{0}=\frac{(n-1)^{(n-1)}}{n^{n}} \frac{1}{c^{\frac{n}{p}}}\left(\frac{\widetilde{L}}{\widetilde{\ell}}\right)^{\frac{\sigma+1}{p}(1-n)}=\tilde{c}_{n, p}\left(\frac{\widetilde{\ell}}{\widetilde{L}}\right)^{\widetilde{\sigma}}
$$

with $\tilde{c}_{n, p}:=\frac{(n-1)^{(n-1)}}{n^{n}} \frac{1}{c^{\frac{n}{p}}}$ and $\widetilde{\sigma}:=\frac{\sigma+1}{p}(n-1)$. Note that $\zeta_{0} \in(0,1)$. In case in which $p \geq 2$, we need

$$
\left(\frac{\beta L}{\tilde{\ell}}\right)^{\frac{2}{p}}<\zeta_{0}
$$

Recalling that $\widetilde{\ell} \geq(\alpha+1) \ell$ and $\widetilde{L} \leq(\beta+1) L$, in order to have (5.17) it suffices to impose that

$$
\left(\frac{\beta}{\alpha+1}\right)^{\frac{2}{p}}\left(\frac{\beta+1}{\alpha+1}\right)^{\tilde{\sigma}}<\tilde{c}_{n, p}\left(\frac{\ell}{L}\right)^{\tilde{\sigma}+\frac{2}{p}} .
$$


In fact

$$
\begin{aligned}
(\beta L)^{\frac{2}{p}}((\beta+1) L)^{\tilde{\sigma}} & <\tilde{c}_{n, p}((\alpha+1) \ell)^{\widetilde{\sigma}+\frac{2}{p}} \Rightarrow(\beta L)^{\frac{2}{p}} \widetilde{L}^{\widetilde{\sigma}}<\tilde{c}_{n, p}(\widetilde{\ell})^{\widetilde{\sigma}+\frac{2}{p}} \\
& \Leftrightarrow\left(\frac{\beta L}{\widetilde{\ell}}\right)^{\frac{2}{p}}<\zeta_{0}=\tilde{c}_{n, p}\left(\frac{\widetilde{\ell}}{\widetilde{L}}\right)^{\tilde{\sigma}}
\end{aligned}
$$

and inequality (5.18) is clearly fulfilled if the ratio $\frac{\beta}{\alpha+1}$ is sufficiently small.

Similarly, in case in which $1<p<2$, we need

$$
\left(\frac{\beta L}{\widetilde{\ell}}\right)\left(\frac{\widetilde{L}}{\widetilde{\ell}}\right)^{\frac{1}{p}}<\zeta_{0}
$$

In order to have (5.19), it suffices to impose

$$
\frac{\beta}{\alpha+1}\left(\frac{\beta+1}{\alpha+1}\right)^{\tilde{\sigma}+\frac{1}{p}}<\tilde{c}_{n, p}\left(\frac{\ell}{L}\right)^{\tilde{\sigma}+1+\frac{1}{p}} .
$$

In fact

$$
\begin{aligned}
\beta(\beta+1)^{\tilde{\sigma}+\frac{1}{p}} L^{\tilde{\sigma}+1+\frac{1}{p}} & <\widetilde{c}_{n, p}((\alpha+1) \ell)^{\tilde{\sigma}+1+\frac{1}{p}} \Rightarrow(\beta L) \widetilde{L}^{\widetilde{\sigma}+\frac{1}{p}}<\tilde{c}_{n, p}(\widetilde{\ell})^{\tilde{\sigma}+1+\frac{1}{p}} \\
& \Leftrightarrow\left(\frac{\beta L}{\widetilde{\ell}}\right)\left(\frac{\widetilde{L}}{\widetilde{\ell}}\right)^{\frac{1}{p}}<\tilde{c}_{n, p}\left(\frac{\widetilde{\ell}}{\widetilde{L}}\right)^{\widetilde{\sigma}}=\zeta_{0}
\end{aligned}
$$

and inequality (5.20) is clearly fulfilled if the ratio $\frac{\beta}{\alpha+1}$ is sufficiently small.

Then, choosing $\alpha, \beta$ such that (5.17) (if $p \geq 2$ ) or (5.19) (if $1<p<2$ ) are satisfied, in view of (5.15) there exist $\vartheta \in\left(0, \vartheta_{0}\right)$ and $\bar{\delta}>0$, depending on $\alpha, \beta, n, p, \ell, L$, such that

$$
\int_{B_{\vartheta r}}|\nabla u|^{p} \mathrm{~d} x \leq \vartheta^{n-1+p \bar{\delta}} \int_{B_{r}}|\nabla u|^{p} \mathrm{~d} x+c(n, p, \mu, \widetilde{L}, \widetilde{\ell}) r^{n} .
$$

Since $r<1$, the term $r^{n}$ can be majorized by $r^{n-1+p \delta}$, for every $0<\delta<\min \left\{\bar{\delta}, \frac{1}{p}\right\}$, and from the previous estimate, we deduce that

$$
\int_{B_{\vartheta r}}|\nabla u|^{p} \mathrm{~d} x \leq \vartheta^{n-1+p \bar{\delta}} \int_{B_{r}}|\nabla u|^{p} \mathrm{~d} x+c(n, p, \mu, \widetilde{L}, \widetilde{\ell}) r^{n-1+p \delta} .
$$

This estimate, by virtue of Lemma 2.4, yields that for all $0<\varrho<r<1$

$$
\int_{B_{\varrho}}|\nabla u|^{p} \mathrm{~d} x \leq c(n)\left(\frac{\varrho}{r}\right)^{n-1+p \delta} \int_{B_{r}}|\nabla u|^{p} \mathrm{~d} x+c \rho^{n-1+p \delta}
$$

So, for $0<\varrho \ll 1$

$$
\int_{B_{\varrho}}|\nabla u|^{p} \mathrm{~d} x \leq c \rho^{n-1+p \delta}
$$

and by Hölder's inequality

$$
\int_{B_{\varrho}}|\nabla u| \mathrm{d} x \leq c\left(\int_{B_{\varrho}}|\nabla u|^{p} \mathrm{~d} x\right)^{\frac{1}{p}} \varrho^{\frac{n}{p^{\prime}}} \leq c \varrho^{\frac{n-1+p \delta+n(p-1)}{p}}=c \rho^{n-1+\delta+\frac{1}{p^{\prime}}} .
$$


By Theorem 2.2, the previous inequality implies that $u \in C_{\mathrm{loc}}^{0, \frac{1}{p^{\prime}}+\delta}(\Omega)$ whenever (5.18) (if $p \geq 2$ ) or (5.20) (if $1<p<2$ ) hold true.

Step 2. Fix a point $x \in \Omega$ and let $\bar{r}>0$ be such that $\operatorname{dist}(x, \partial \Omega)>\bar{r}$. Consider $0<r<r_{0} \leq \bar{r}$ and denote by $A$ any set of finite perimeter such that $E \Delta A \subset \subset B_{r}(x)$. From Theorem 1.4 we have that

$$
\mathcal{I}_{\lambda_{0}}(u, E) \leq \mathcal{I}_{\lambda_{0}}(u, A)
$$

and thus

$$
\begin{aligned}
& \int_{\Omega}\left(F(\nabla u)+\chi_{E} G(\nabla u)\right) \mathrm{d} x+P(E, \Omega)+\lambda_{0}|| E|-d| \\
\leq & \int_{\Omega}\left(F(\nabla u)+\chi_{A} G(\nabla u)\right) \mathrm{d} x+P(A, \Omega)+\lambda_{0}|| A|-d| .
\end{aligned}
$$

Using that $E \Delta A \subset \subset B_{r}(x)$, we deduce that

$$
\begin{aligned}
P\left(E, B_{r}(x)\right)-P\left(A, B_{r}(x)\right) & \leq \int_{B_{r}}\left(\chi_{A}(x)-\chi_{E}(x)\right) G(\nabla u) \mathrm{d} x+\lambda_{0}|| A|-| E|| \\
& \leq \beta L \int_{B_{r}}|\nabla u|^{p}+c r^{n},
\end{aligned}
$$

where we invoked assumption (G2). By the decay estimate (5.21), we infer that

$$
P\left(E, B_{r}(x)\right)-P\left(A, B_{r}(x)\right) \leq c r^{n-1+p \delta}+c r^{n} \leq c r^{n-1+p \delta}
$$

since $r<1$. As $\delta$ can be replaced by any smaller number, we can choose $p \delta<\frac{1}{2}$ and the result follows from Theorem 2.3.

\section{Partial Regularity - Proof of Theorem 1.3}

In this section, we prove that a partial regularity result holds without imposing any bounds on $\alpha$ and $\beta$, as stated in Theorem 1.3.

Proof of Theorem 1.3. Set

$$
\Omega_{0}:=\left\{x \in \Omega: \limsup _{\rho \rightarrow 0} \frac{1}{\rho^{n-1+p \delta}} \int_{B_{\rho}}|\nabla u|^{p}=0\right\},
$$

for an arbitrary $0<\delta<\frac{1}{p}$. Note that (see Thm. 3, Sect. 2.4.3, in [9]), $\left|\Omega \backslash \Omega_{0}\right|=0$. Fix a point $x \in \Omega_{0}$ and let $r_{0}$ be such that $\operatorname{dist}(x, \partial \Omega)>r_{0}$. Since $x \in \Omega_{0}$, for every $\varepsilon>0$ there exists a radius $R=R(\varepsilon)<r_{0}$ such that

$$
\frac{1}{r^{n-1+p \delta}} \int_{B_{r}}|\nabla u|^{p}<\varepsilon
$$

for all $0<r \leq R(\varepsilon)$. By (5.15) and (5.17), for all $0<\varrho<r$ we have

$$
\int_{B_{\varrho}}|\nabla u|^{p} \mathrm{~d} x \leq c_{n, p}\left[\zeta+\left(\frac{\widetilde{L}}{\widetilde{\ell}}\right)^{\frac{\sigma+1}{p}}\left(\frac{\rho}{r}\right)^{n / p}\right]^{p} \int_{B_{r}}|\nabla u|^{p} \mathrm{~d} x+c(n, p, \widetilde{L}, \tilde{\ell}, \mu) r^{n} .
$$

Inserting (6.1) in previous inequality, we get

$$
\int_{B_{\varrho}}|\nabla u|^{p} \mathrm{~d} x \leq c \varepsilon^{\frac{1}{2}}\left[1+\left(\frac{\varrho}{r}\right)^{n / p}\right]^{p} r^{\frac{n-1+p \delta}{2}}\left(\int_{B_{r}}|\nabla u|^{p} \mathrm{~d} x\right)^{\frac{1}{2}}+c r^{n},
$$


where $c=c(n, p, \mu, \alpha, \beta, \ell, L)$. By Young's inequality, we deduce that

$$
\begin{aligned}
\int_{B_{\varrho}}|\nabla u|^{p} \mathrm{~d} x & \leq c \varepsilon^{\frac{1}{2}}\left[1+\left(\frac{\varrho}{r}\right)^{n / p}\right]^{p}\left\{\int_{B_{r}}|\nabla u|^{p} \mathrm{~d} x+r^{n-1+p \delta}\right\}+c r^{n} \\
& \leq c \varepsilon^{\frac{1}{2}}\left[1+\left(\frac{\varrho}{r}\right)^{n / p}\right]^{p} \int_{B_{r}}|\nabla u|^{p} \mathrm{~d} x+c r^{n-1+p \delta} .
\end{aligned}
$$

for every $0<\rho<r \leq R(\varepsilon)$, since we may suppose, without loss of generality, that $r<1$. Therefore, in particular, writing (6.2) for $\rho=\frac{r}{2}$, we get

$$
\int_{B_{\frac{r}{2}}}|\nabla u|^{p} \mathrm{~d} x \leq c \varepsilon^{\frac{1}{2}}\left[1+\left(\frac{1}{2}\right)^{n / p}\right]^{p} \int_{B_{r}}|\nabla u|^{p} \mathrm{~d} x+c r^{n-1+p \delta} .
$$

Choosing $\varepsilon$ in $(6.3)$ such that

$$
\varepsilon^{\frac{1}{2}}<\frac{2^{1-p}}{c\left(1+2^{\frac{n}{p}}\right)^{p}}
$$

we obtain

$$
\begin{aligned}
\int_{B_{\frac{r}{2}}}|\nabla u|^{p} \mathrm{~d} x & \leq \frac{2^{1-p}}{\left(1+2^{\frac{n}{p}}\right)^{p}}\left[1+\left(\frac{1}{2}\right)^{n / p}\right]^{p} \int_{B_{r}}|\nabla u|^{p} \mathrm{~d} x+c r^{n-1+p \delta} \\
& =\frac{2^{1-p}}{\left(1+2^{\frac{n}{p}}\right)^{p}} \frac{\left(1+2^{\frac{n}{p}}\right)^{p}}{2^{n}} \int_{B_{r}}|\nabla u|^{p} \mathrm{~d} x+c r^{n-1+p \delta} \\
& =\left(\frac{1}{2}\right)^{n-1+p} \int_{B_{r}}|\nabla u|^{p} \mathrm{~d} x+c r^{n-1+p \delta} .
\end{aligned}
$$

From (6.4), thanks to Lemma 2.4 applied with $\varphi(r):=\int_{B_{r}}|\nabla u|^{p} \mathrm{~d} x$ and $\vartheta=\frac{1}{2}$, we obtain that

$$
\int_{B_{\varrho}}|\nabla u|^{p} \mathrm{~d} x \leq c \rho^{n-1+p \delta}
$$

for all $0<\varrho<r<r_{0}$ and some $c=c(n, p, \alpha, \beta, \ell, L)$. Hence, by virtue of Theorem 2.2 and Hölder inequality, we deduce that $u \in C^{0, \frac{1}{p^{\prime}}+\delta}\left(\Omega_{0}\right)$, for every $0<\delta<\frac{1}{p}$.

Let us denote by $A$ any set of finite perimeter such that $E \Delta A \subset \subset B_{\rho}(x)$. From Theorem 1.4 we have that

$$
\mathcal{I}_{\lambda_{0}}(u, E) \leq \mathcal{I}_{\lambda_{0}}(u, A),
$$

therefore, by assumption (G1) and the decay estimate (6.5), we deduce that

$$
\begin{aligned}
P\left(E, B_{\rho}(x)\right)-P\left(A, B_{\rho}(x)\right) & \leq \int_{B_{\rho}}\left(\chi_{A}(x)-\chi_{E}(x)\right) G(\nabla u) \mathrm{d} x+\lambda_{0}|| A|-| E|| \\
& \leq \beta L \int_{B_{\rho}}|\nabla u|^{p} \mathrm{~d} x+\lambda_{0}|| A|-| E|| \leq c \rho^{n-1+p \delta}+c \lambda_{0} \rho^{n}
\end{aligned}
$$

and the conclusion follows again by Theorem 2.3 applied to $\Omega_{0}$ in place of $\Omega$.

Acknowledgements. The authors warmly thank the Center for Nonlinear Analysis (NSF Grants Nos. DMS- 0405343 and DMS-0635983), where part of this research was carried out. The research of I. Fonseca was partially funded by the National Science Foundation under Grant No. DMS- 0905778. 


\section{REFERENCES}

[1] H.W. Alt and L.A. Caffarelli, Existence and regularity results for a minimum problem with free boundary. J. Reine Angew. Math. 325 (1981) 107-144.

[2] E. Acerbi and N. Fusco, Regularity for minimizers of non-quadratic functionals: the case $1<p<2$. J. Math. Anal. Appl. 140 (1989) 115-135.

[3] E. Acerbi and N. Fusco, A regularity theorem for minimizers of quasi-convex integrals. Arch. Rational Mech. Anal. 99 (1987) 261-281.

[4] L. Ambrosio and G. Buttazzo, An optimal design problem with perimeter penalization. Calc. Var. Partial Differ. Eq. 1 (1993) $55-69$.

[5] L. Ambrosio, N. Fusco and D. Pallara, Functions of Bounded Variation and Free Discontinuity Problems. Oxford University Press (2000).

[6] E. Bombieri, Regularity theory for almost minimal currents. Arch. Rational Mech. Anal. 78 (1982) 99-130.

[7] M. Carozza and A. Passarelli Di Napoli, A regularity theorem for minimisers of quasiconvex integrals: The case $1<p<2$. Proc. Roy. Soc. Edinburgh A Math. 126, 6 (1996) 1181-1200.

[8] L. Esposito and N. Fusco, A remark on a free interface problem with volume constraint. J. Convex Anal. 18 (2011) $417-426$.

[9] L.C. Evans and F.R. Gariepy, Measure theory and fine properties of functions. Studies in Advanced Mathematics. CRC Press, Boca Raton, FL (1992).

[10] I. Fonseca and N. Fusco, Regularity results for anisotropic image segmentation models. Ann. Sci. Norm. Super. Pisa 24 (1997) 463-499.

[11] I. Fonseca, N. Fusco, G. Leoni and V. Millot, Material voids in elastic solids with anisotropic surface energies. J. Math. Pures Appl. 96 (2011).

[12] I. Fonseca, N. Fusco, G. Leoni and M. Morini, Equilibrium configurations of epitaxially strained crystalline films: existence and regularity results. Arch. Rational Mech. Anal. 186 (2007) 477-537.

[13] N. Fusco and J. Hutchinson, $C^{1, \alpha}$ partial regularity of functions minimising quasiconvex integrals. Manuscripta Math. 54 (1985) 121-143.

[14] M. Giaquinta, Multiple integrals in the calculus of variations and nonlinear ellyptic systems. Ann. Math. Stud. Princeton University Press (1983).

[15] M. Giaquinta and G. Modica, Partial regularity of minimizers of quasiconvex integrals. Ann. Inst. Henri Poincaré, Anal. Non Linéaire 3 (1986) 185-208.

[16] D. Gilbarg and N.S. Trudinger, Elliptic partial differential equations of second order, 2nd edn., vol. 224 of Grundlehren der Mathematischen Wissenschaften. Springer-Verlag, Berlin (1983).

[17] E. Giusti. Direct methods in the calculus of variations. World Scientific (2003).

[18] M. Gurtin, On phase transitions with bulk, interfacial, and boundary energy. Arch. Rational Mech. Anal. 96 (1986) 243-264

[19] C.J. Larsen, Regularity of components in optimal design problems with perimeter penalization. Calc. Var. Partial Differ. Eq. 16 (2003) 17-29.

[20] F.H. Lin, Variational problems with free interfaces. Calc. Var. Partial Differ. Eq. 1 (1993) 149-168.

[21] F.H. Lin and R.V. Kohn, Partial regularity for optimal design problems involving both bulk and surface energies. Chin. Ann. Math. B 20, (1999) 137-158.

[22] V. Šverák and X. Yan. Non-Lipschitz minimizers of smooth uniformly convex variational integrals. Proc. Natl. Acad. Sci. USA 99 (2002) 15269-15276.

[23] I. Tamanini, Boundaries of Caccioppoli sets with Hölder-continuous normal vector. J. Reine Angew. Math. 334 (1982) 27-39. 